ACCepted by ApJ (February 8, 2010)

Preprint typeset using $\mathrm{LAT}_{\mathrm{E}} \mathrm{X}$ style emulateapj v. 05/04/06

\title{
NUCLEOSYNTHESIS IN TWO-DIMENSIONAL DELAYED DETONATION MODELS OF TYPE IA SUPERNOVA EXPLOSIONS
}

\author{
K. MAeda ${ }^{1,2}$, F.K. RÖPKE ${ }^{2}$, M. Fink ${ }^{2}$, W. HillebrandT ${ }^{2}$, \\ C. Travaglio ${ }^{2,3}$, F.-K. Thielemann ${ }^{4,5}$ \\ Accepted by ApJ (February 8, 2010)
}

\begin{abstract}
For the explosion mechanism of Type Ia supernovae (SNe Ia), different scenarios have been suggested. In these, the propagation of the burning front through the exploding white dwarf star proceeds in different modes, and consequently imprints of the explosion model on the nucleosynthetic yields can be expected. The nucleosynthetic characteristics of various explosion mechanisms is explored based on three two-dimensional explosion simulations representing extreme cases: a pure turbulent deflagration, a delayed detonation following an approximately spherical ignition of the initial deflagration, and a delayed detonation arising from a highly asymmetric deflagration ignition. Apart from this initial condition, the deflagration stage is treated in a parameter-free approach. The detonation is initiated when the turbulent burning enters the distributed burning regime. This occurs at densities around $10^{7} \mathrm{~g} \mathrm{~cm}^{-3}$ - relatively low as compared to existing nucleosynthesis studies for one-dimensional spherically symmetric models. The burning in these multidimensional models is different from that in one-dimensional simulations as the detonation wave propagates both into unburned material in the high density region near the center of a white dwarf and into the low density region near the surface. Thus, the resulting yield is a mixture of different explosive burning products, from carbonburning products at low densities to complete silicon-burning products at the highest densities, as well as electron-capture products synthesized at the deflagration stage. Detailed calculations of the nucleosynthesis in all three models are presented. In contrast to the deflagration model, the delayed detonations produce a characteristic layered structure and the yields largely satisfy constraints from Galactic chemical evolution. In the asymmetric delayed detonation model, the region filled with electron capture species (e.g., ${ }^{58} \mathrm{Ni},{ }^{54} \mathrm{Fe}$ ) is within a shell, showing a large off-set, above the bulk of ${ }^{56} \mathrm{Ni}$ distribution, while species produced by the detonation are distributed more spherically.

Subject headings: nuclear reactions, nucleosynthesis, abundances - hydrodynamics - supernovae: general
\end{abstract}

\section{INTRODUCTION}

There is a consensus that Type Ia supernovae ( $\mathrm{SNe}$ Ia) are the outcome of a thermonuclear explosion of a carbon-oxygen white dwarf (WD) (e.g., Wheeler et al. 1995; Nomoto et al. 1997; Branch 1998). For the progenitor, the Chandrasekhar-mass $\left(M_{\mathrm{Ch}}\right)$ WD model has been favored for a majority of SNe Ia (e.g., Höflich \& Khokhlov 1996; Nugent et al. 1997; Fink et al. 2007; Mazzali et al. 2007).

The mass of the WD could reach $M_{\mathrm{Ch}}$ by several evolutionary paths, either by a mass-transfer from a binary giant/main-sequence companion (single degenerate scenario; e.g., Whelan \& Iben 1973; Nomoto 1982) or as a result of merging with a binary degenerate WD companion (double degenerate scenario; e.g., Iben \& Tutukov 1984; Webbink 1984). As the WD has accreted a sufficient amount of material, the central density of the WD increases and the heating rate by the carbon fusion ex-

\footnotetext{
${ }^{1}$ Institute for the Physics and Mathematics of the Universe (IPMU), University of Tokyo, 5-1-5 Kashiwanoha, Kashiwa, Chiba 277-8583, Japan; keiichi.maeda@ipmu.jp.

2 Max-Planck-Institut für Astrophysik, Karl-SchwarzschildStraße 1, 85741 Garching, Germany.

3 INAF-Osservatorio Astronomico di Torino, Strada dell'Osservatorio 20, I-10025 Pino Torinese, Torino, Italy

${ }^{4}$ Department Physik, Universität Basel, CH-4056 Basel, Switzerland

${ }^{5}$ GSI Helmholtz Center, Planckstr. 1, 64291 Darmstadt, Germany
}

ceeds the cooling rate by neutrino emission. The evolution is then followed by a simmering phase with convective carbon burning, lasting for about a century. At the end of this simmering phase, the temperature rises to the point where convection can no longer efficiently transport away the energy produced by the carbon burning. This is a stage where the burning becomes dynamical, initiating a thermonuclear flame that propagates outward and disrupts the WD, i.e., a supernova explosion (Nomoto et al. 1984; Woosley \& Weaver 1986).

Once the thermonuclear flame is ignited, there are two possible modes of the propagation: subsonic deflagration and supersonic detonation. A prompt ignition of the detonation flame is disfavored because the resulting nucleosynthesis yield conflicts with Galactic chemical evolution (Arnett 1969) and fails to produce the strong intermediate-mass element features observed in SNe Ia. Thus, the explosion should start with a subsonic deflagration flame. The deflagration stage may last until the end of the explosion (the deflagration model; Nomoto et al. 1984), while it is also possible that the deflagration flame turns into the detonation wave [the delayed detonation model, or the deflagration-detonation transition (DDT) model; Khokhlov 1991; Yamaoka et al. 1992; Woosley \& Weaver 1994; Iwamoto et al. 1999].

The supernova explosion phase has been investigated by "classical" one-dimensional spherically symmetric models; the classical deflagration W7 model of Nomoto 
et al. (1984) has successfully explained the basic contribution of SNe Ia to Galactic chemical evolution, as well as basic features of observed spectra and light curves of individual SNe Ia of a normal class (Branch et al. 1985). Some improvement in these observational aspects has been obtained by introducing a delayed detonation (e.g., Höflich \& Khokhlov 1996; Iwamoto et al. 1999).

These models, however, treat the propagation speed of the deflagration flame as a parameter. Moreover, the deflagration flame is hydrodynamically unstable and nonsphericity is thus actually essential (e.g., Niemeyer et al. 1996). Recent investigations of the explosion models have intensively addressed these issues (e.g., Reinecke et al. 2002; Gamezo et al. 2003; Röpke \& Hillebrandt 2005; Bravo \& García-Senz 2006; Röpke et al. 2006b; Schmidt \& Niemeyer 2006). With high resolution multidimensional hydrodynamic simulations, coupled with an appropriate sub-grid model to capture turbulence effects on unresolved scales, recent studies provide essentially "parameter-free" simulations for the initial deflagration stage, where only the structure of the pre-supernova WD and the distribution of the initial deflagration ignition sparks set up the initial conditions.

The multi-dimensional simulations have been performed with different initial conditions to see if SNe Ia in general can be explained in a framework of a pure deflagration explosion, as is summarized by Röpke et al. (2007b). Detailed nucleosynthesis calculations have been performed for some deflagration models (Travaglio et al. 2004a, 2005; Kozma et al. 2005, Röpke et al. 2006a). These studies indicate that the pure deflagration explosion can explain a part of SNe Ia, up to relatively weak and faint SNe Ia in the normal population. However, it has also been shown that a subsequent detonation phase is probably necessary to account for typical normal SNe Ia and brighter ones (Gamezo et al. 2005; Röpke et al. 2007b).

Delayed detonation models have also been investigated with multi-dimensional simulations (Gamezo et al. 2005; Plewa 2007; Golombek \& Niemeyer 2005; Röpke \& Niemeyer 2007c; Bravo \& García-Senz 2008). However, compared to the deflagration models as summarized above, the investigation of multi-dimensional delayed detonation models is still at the very initial stage. The radiation transfer based on the multi-dimensional models has been examined by Kasen et al. (2009). Detailed nucleosynthesis studies have rarely been done (but see Bravo \& García-Senz 2008). In this paper, we present results from detailed nucleosynthesis calculations, based on two-dimensional delayed detonation models and, for comparison, a pure deflagration model.

The two delayed detonation models presented here can be regarded as extreme cases - not necessarily with respect to their ${ }^{56} \mathrm{Ni}$ production and brightness, but with respect to symmetries/asymmetries in the explosion phase. While in one model, the deflagration was ignited in an approximately spherical configuration at the center of the WD, the other model features an off-center ignition and propagation of the initial deflagration flame similar to the three-dimensional simulations of Röpke et al. (2007d). It has been suggested that the convection in the simmering phase may be dominated by a dipolar mode, and there is a good possibility that the deflagration is initiated in an off-center way (e.g., Woosley et al. 2004, Kuhlen et al. 2006). In the pure-deflagration model, such an explosion cannot account for normal SNe Ia, because the small burning surface area should result in inefficient production of ${ }^{56} \mathrm{Ni}$ (see, e.g., Röpke et al. 2007d). However, this can be possibly overcome in the delayed detonation scenario, as the detonation can potentially produce a large amount of ${ }^{56} \mathrm{Ni}$.

The paper is organized as follows. In $\S 2$, we present methods and models, in $\S 3$, we present our results. Discussion of these results from a view point of the chemical evolution is given in $\S 4$. The paper is closed in $\S 5$ with concluding remarks.

\section{METHODS AND MODELS}

\subsection{Explosion Models}

In this paper, we concentrate on two-dimensional models. Although some results might be affected by the imposed symmetry (e.g., Travaglio et al. 2004a; Röpke et al. $2007 \mathrm{~b}$ ), we believe that examining 2D models is a natural step forward. Moreover, it illustrates how nucleosynthesis in the different models proceeds, and highlights differences with respect to one-dimensional models.

In particular, we focus on three models in this paper as follows:

- C-DEF: A globally spherically symmetric puredeflagration explosion in which the deflagration was ignited within the c3-shape boundary, as was done by Reinecke et al. (1999a). About $2 \times$ $10^{-2} M_{\odot}$ were initially incinerated to trigger the deflagration. This model is similar to one presented already in Travaglio et al. (2004a).

- C-DDT: A delayed detonation model which follows the 2D spherical deflagration C-DEF model. A prescription for the DDT is given below.

- O-DDT: A delayed detonation model which follows an extremely off-center deflagration. The deflagration is ignited by 29 bubbles distributed within an opening angle with respect to the $z$-axis of 45 degrees. The outermost bubble was placed at a distance of $\sim 180 \mathrm{~km}$ from the center. About $1 \times 10^{-5} M_{\odot}$ were initially incinerated to trigger the deflagration. The DDT is treated in the same way as in the C-DDT model.

The exploding WD had a central density of $2.9 \times$ $10^{9} \mathrm{~g} \mathrm{~cm}^{-3}$. In the hydrodynamic explosion simulations, burning was treated in a simplified way. Only five species (carbon, oxygen, alpha-particles, a representative for iron-peak elements, and a representative for intermediate mass elements) were followed. The deflagration and detonation fronts were modeled with the level-set approach (Reinecke et al. 1999b; Golombek \& Niemeyer 2005; Roepke \& Niemeyer 2007c). After passage of the zero-level set representing the combustion waves, the material was converted from the carbon/oxygen fuel mixture to an approximate nuclear ash composition. At high densities, NSE is reached and the ash was modeled as a temperature- and density-dependent mixture of ironpeak elements and alpha-particles. In the NSE region, electron captures neutronizing the ashes were followed. In the hydrodynamic simulations, $512 \times 512$ cells are used 
TABLE 1

$\operatorname{MASS}\left(M_{\odot}\right)$ AND ENERGETIC $\left(10^{51}\right.$ ERG $)$

\begin{tabular}{|c|c|c|c|c|c|c|}
\hline Form & W7 & C-DEF & C-DDT & O-DDT & C-DDT/def. ${ }^{a}$ & O-DDT/def. ${ }^{a}$ \\
\hline$M_{\text {burn }}{ }^{\mathrm{b}}$ & 1.21 & 0.61 & 1.01 & 1.25 & 0.522 & 0.393 \\
\hline$E_{\text {nuc }}{ }^{c}$ & 1.78 & 0.91 & 1.46 & 1.80 & 0.767 & 0.522 \\
\hline$E_{\mathrm{K}}^{\mathrm{d}}$ & 1.28 & 0.41 & 0.96 & 1.30 & $\ldots$ & $\ldots$ \\
\hline$E_{\mathrm{K}}(\text { hyd })^{\mathrm{e}}$ & 1.30 & 0.41 & 0.93 & 1.27 & $\ldots$ & $\ldots$ \\
\hline
\end{tabular}

${ }^{\mathrm{a}}$ At the moment when the first DDT takes place.

$\mathrm{b}_{\text {The incinerated masses. }}$

${ }^{\mathrm{c}}$ The nuclear energy generation derived by the postprocess calculations.

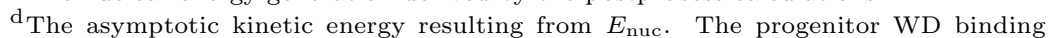
energy is $E_{\text {bind }}=0.505$.

The asymptotic kinetic energy calculated in the hydrodynamic simulations with the simplified treatment of the energy generation.

for C-DEF and C-DDT models, and $1024 \times 512$ cells are used for O-DDT model.

In the delayed detonation models, we assumed the deflagration-to-detonation transition to take place once the flame enters the distributed burning regime (e.g., Röpke \& Niemeyer 2007c) at a fuel density of $\leq 1 \times$ $10^{7} \mathrm{~g} \mathrm{~cm}^{-3}$ (hereafter $\rho_{\text {DDT }}$ ). We emphasize that in contrast to the classical 1D models, the DDT is not exclusively parameterized by $\rho_{\mathrm{DDT}}$, but the requirement on reaching the distributed burning implies also a turbulence criterion (see Röpke 2007a for an evaluation in three-dimensional models). An estimate for entering the distributed regime is the equality of the laminar flame width and the Gibson scale - the scale at which the turbulent velocity fluctuations equal the laminar flame speed. When the Gibson scale becomes smaller than the laminar flame width, turbulence affects (and ultimately destroys) the laminar flame structure. This is a prerequisite for DDT, however, it is not a sufficient criterion (e.g. Woosley 2007; Woosley et al. 2009). The microphysics of DDT is not yet fully known, and therefore we apply the necessary condition of entering the distributed regime here only. We emphasize that the hydrodynamic models are still in a preliminary stage and used here only in order to demonstrate the nucleosynthesis associated with them. For more robust predictions of the ${ }^{56} \mathrm{Ni}$ production and the implied brightness of the events, more elaborate hydrodynamical simulations should be used (F.K. Röpke et al., in prep.); the models presented here are understood as a case study demonstrating typical nucleosynthesis for the different explosion processes.

Results on some synthetic observables (e.g., light curves) derived for similar two-dimensional models can be found in Kasen et al. (2009), and details on hydrodynamic calculations will be presented elsewhere (F.K. Röpke et al., in prep.). Note that the present models cover extreme cases in the sequence of "classical" delayed detonation models where the DDT takes place before the deflagration wave reaches the WD surface (e.g., Kasen et al. 2009). For the situation in which only a few initial bubbles are distributed within a small solid angle with a large off-set, the "gravitationally confined detonation" model (e.g. Jordan et al. 2008; Meakin et al. 2009) has been suggested as an alternative explosion mechanism. This would be a more extreme case than our O-DDT model.

\subsection{Nucleosynthesis}

We apply the tracer particle method to the calculations of nucleosynthesis. The essence is to follow thermal histories of Lagrangian particles, which are passively advected in hydrodynamic simulations, and then to employ detailed nuclear reaction network calculations to each particle separately. The method was first applied to corecollapse supernovae (Nagataki et al. 1997; Maeda et al. 2002; Maeda \& Nomoto 2003), and has become popular in the field thanks to its simplicity and its applicability to multi-dimensional problems (e.g., Travaglio et al. 2004b). Travaglio et al. (2004a) applied the method to multi-dimensional, purely deflagration explosion models of SNe Ia.

In the setup for our hydrodynamic simulations, $80^{2}$ tracer particles are distributed uniformly in mass coordinate, such that each particle represents the same mass of $\sim 2.2 \times 10^{-4} M_{\odot}\left(=M_{\mathrm{wd}} / 6400\right)$. The particles are advected passively, following the velocity field at each time step of the Eulerian hydrodynamic simulations. The thermal history each particle experiences is recorded. The number of the tracer particles is sufficient to accurately follow the nucleosynthesis. Seitenzahl et al. (in preparation) find that in two-dimensional SN Ia simulations with $80^{2}$ tracer particles all isotopes with abundances higher than $\sim 10^{-5}$ are reproduced with an accuracy of better than $5 \%$ (except for ${ }^{20} \mathrm{Ne}$ ).

The nuclear postprocessing calculations are then performed for each particle separately. To this end, we recalculate the temperature from the recorded internal energy, rather than directly using the value obtained by the hydrodynamic simulations (see Travaglio et al. 2004a). In deriving the temperature, the electron fraction $\left(Y_{\mathrm{e}}\right)$ is assumed to be 0.5 , which introduces some errors when the electron captures are very active. When $T_{9}=T / 10^{9} \mathrm{~K}$ $>6$, we follow the abundance evolution by applying the Nuclear Statistical Equilibrium (NSE) abundance (i.e., the abundance specified by $\rho, T$, and $Y_{\mathrm{e}}$ ) rather than fully solving the reaction network, as the NSE is reached in this regime of high density and temperature. In order to correctly follow the evolution of $Y_{\mathrm{e}}$, weak interactions are computed along with the NSE abundance.

Throughout this paper, the initial $\mathrm{C}+\mathrm{O}$ WD composition is assumed as follows: $\mathrm{X}\left({ }^{12} \mathrm{C}\right)=0.475, \mathrm{X}\left({ }^{16} \mathrm{O}\right)$ $=0.5$, and $\mathrm{X}\left({ }^{22} \mathrm{Ne}\right)=0.025$ in mass fractions. This is consistent with the compositions used in the W7 model, roughly corresponding to the initially solar $\mathrm{CNO}$ composition: Metallicity is represented by ${ }^{22} \mathrm{Ne}$, assuming that the $\mathrm{CNO}$ cycle in the H-burning has converted all heavy elements to ${ }^{14} \mathrm{~N}$, and then it is reprocessed to ${ }^{22} \mathrm{Ne}$ by 

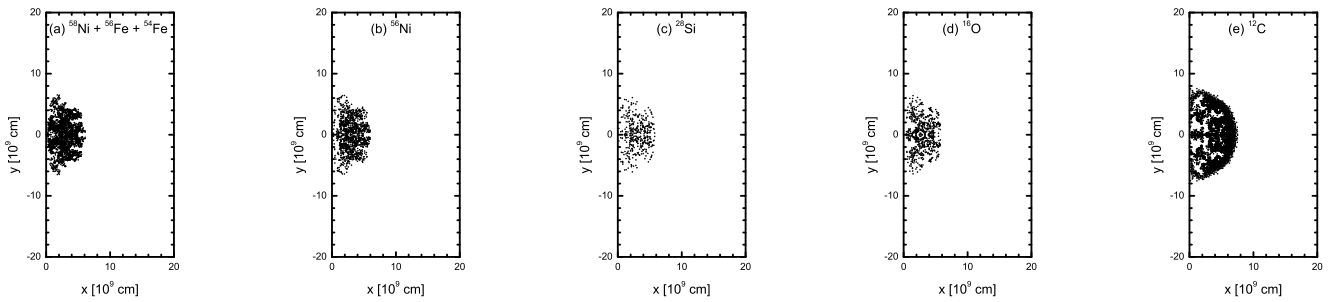

FIG. 1.- Distribution of characteristic elements/isotopes in the C-DEF model, at 10 seconds after the ignition of the deflagration wave. (a) Tracer particles in which the total mass fraction of stable ${ }^{56} \mathrm{Fe},{ }^{58} \mathrm{Ni}$, and ${ }^{54} \mathrm{Fe}$ exceeds 0.1 (electron capture products). (b) Tracer particles in which the mass fraction of ${ }^{56} \mathrm{Ni}$ exceed 0.1, excluding particles shown in (a) (complete Si burning). (c) Tracer particles in which the mass fraction of ${ }^{28} \mathrm{Si}$ exceeds 0.1 and that of ${ }^{16} \mathrm{O}$ is below 0.1 , excluding particles in (a) and (b) (O burning). (d) Tracer particles in which the mass fraction of ${ }^{16} \mathrm{O}$ exceeds 0.1 and that of ${ }^{12} \mathrm{C}$ is below 0.1 , excluding particles in (a) - (c) (C burning). (e) Tracer particles with the mass fraction of ${ }^{12} \mathrm{C}$ exceeding 0.1 , excluding particles in (a) - (d) (no-burning).
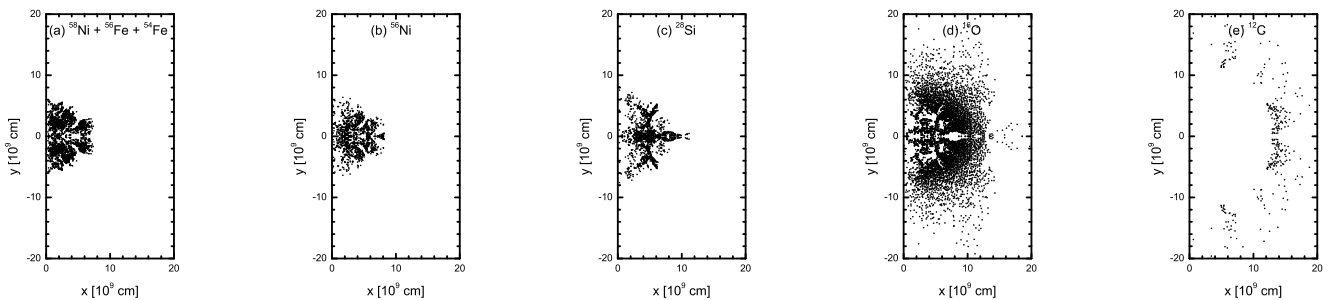

FIG. 2.- Distribution of characteristic elements/isotopes in the C-DDT model, at 10 seconds after the ignition of the deflagration wave. See the caption of Fig. 1.
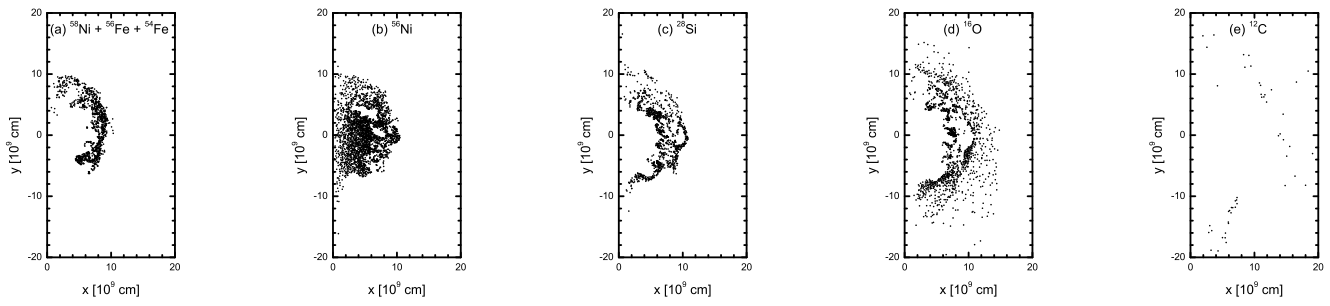

FIG. 3.- Distribution of characteristic elements/isotopes in the O-DDT model, at 10 seconds after the ignition of the deflagration wave. See the caption of Fig. 1.

${ }^{14} \mathrm{~N}(\alpha, \gamma){ }^{18} \mathrm{~F}\left(\mathrm{e}^{+}, \nu_{\mathrm{e}}\right){ }^{18} \mathrm{O}(\alpha, \gamma){ }^{22} \mathrm{Ne}$ in the He-burning.

The reaction network (Thielemann et al. 1996) includes 384 isotopes up to ${ }^{98}$ Mo. The electron capture rates, which strongly affect nucleosynthesis at the beginning of the deflagration stage, are taken from Langanke \& Martinez-Pinedo (2000) and Martinez-Pinedo et al. (2000). Detailed comparisons between the new rates and those of Fuller et al. $(1982,1985)$ are presented by Brachwitz et al. (2000) for the spherical 1D deflagration model W7 (see also Thielemann et al. 2004).

\section{RESULTS}

\subsection{Characteristic Burning Regimes}

Before presenting the result of our calculations, we summarize the basics of explosive burning taking place in the deflagration and detonation stages. Typical burning products can be characterized by the maximum temperatures $\left(T_{\max }\right)$ and densities $\left(\rho_{\max }\right)$ attained by the material under consideration, after the passage of the thermonuclear flame [see, e.g., Arnett (1996), Thielemann et al. (1998), and references therein, for a review of explosive nucleosynthesis].

The explosive burning in thermonuclear supernovae proceeds in different regimes, mainly characterized by the temperatures reached in the nuclear ashes (Thielemann et al. 1986). In deflagrations, this directly translates into characteristic fuel densities ahead of the flame. At the first stage of the deflagration, the density is higher than $10^{8} \mathrm{~g} \mathrm{~cm}^{-3}$. Temperatures rise to $T_{\max , 9} \equiv T_{\max } / 10^{9} \mathrm{~K}$ $\gtrsim 6$. At this temperature, NSE applies, and thus the final composition is determined by freezeout processes (represented by $T_{\max }$ and $\rho_{\max }$ ) and the efficiency of electron capture reactions. Because of the high density, electron capture reactions are important. Dominant species in this "complete silicon burning with electron capture region" are stable ${ }^{56} \mathrm{Fe},{ }^{54} \mathrm{Fe},{ }^{58} \mathrm{Ni}$, and radioactive ${ }^{56} \mathrm{Ni}$ (which decays into ${ }^{56} \mathrm{Fe}$ ). In the case of even stronger electron capture reactions, the main products are ${ }^{50} \mathrm{Ti}$, ${ }^{54} \mathrm{Cr}$, and ${ }^{58} \mathrm{Fe}$ (e.g., Thielemann et al. 2004).

Following the expansion of the WD, the deflagration 

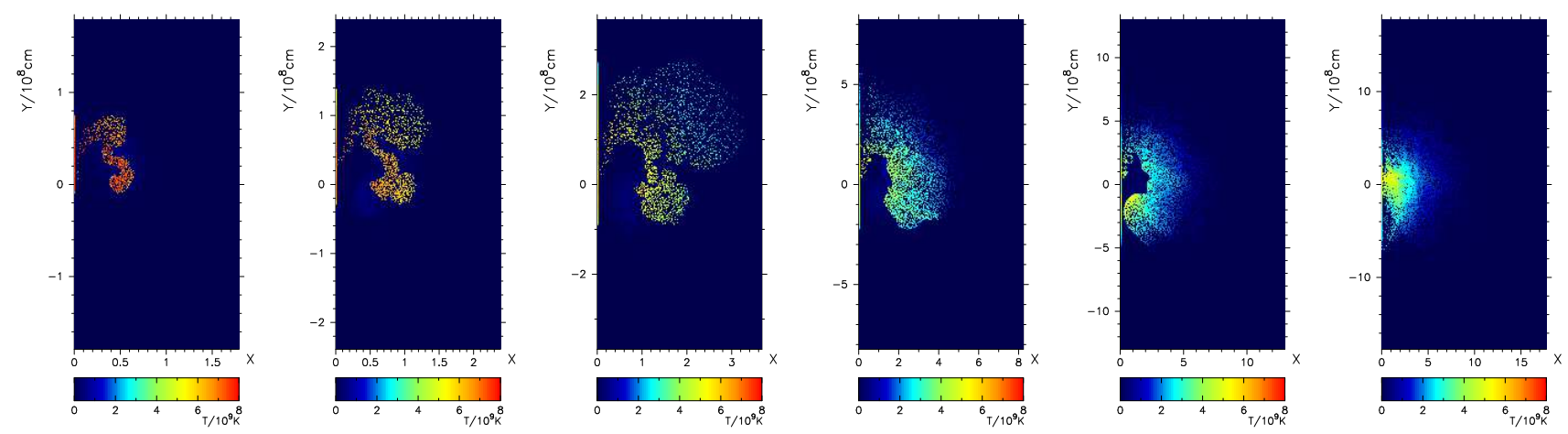

FIG. 4.- Temporal evolution of temperature distribution (at 0.7, 0.9, 1.1, 1.3, 1.5, 1.7 seconds after the ignition of the deflagration flame, from left to right), in the O-DDT model. The color coordinate corresponds to $T_{9}=8$ (red) and 0 (blue). The complete silicon burning takes place at the temperature above $T_{9}=5$ (yellow).
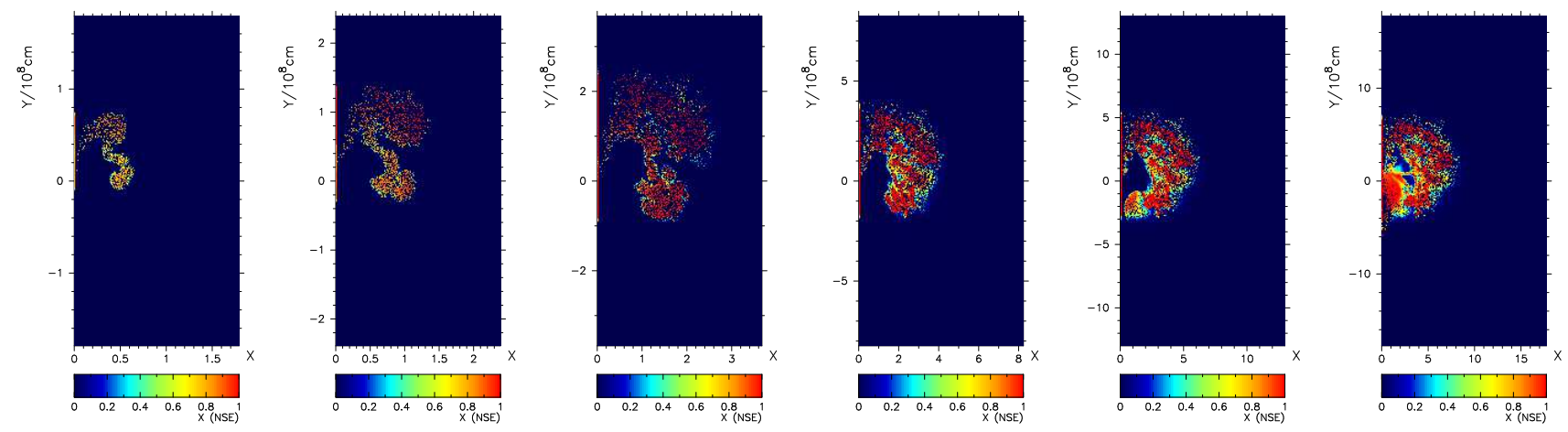

FIG. 5.- Temporal evolution of distribution of NSE elements (at 0.7, 0.9, 1.1, 1.3, 1.5, 1.7 seconds after the ignition of the deflagration flame, from left to right), in the O-DDT model. Shown here is the mass fraction, from 0 (blue) to 1 (red).

proceeds progressively at the lower density. At $T_{\max , 9} \sim$ $5-6$ and $\rho_{\max } \sim 5 \times 10^{7}-10^{8} \mathrm{~g} \mathrm{~cm}^{-3}$, NSE still applies. The electron captures are no longer important, and thus the initial $Y_{\mathrm{e}}$ is virtually preserved. The dominant species are ${ }^{56} \mathrm{Ni}$ and ${ }^{58} \mathrm{Ni}$.

In contrast to spherically-symmetric delayed detonation models, the detonation in our two-dimensional setups is triggered at certain spots at the flame front, but at other locations, deflagration burning can still proceed for some while. It is characterized by successively lower temperature and density. At $T_{\max , 9} \sim 3-5$, oxygen burning or incomplete silicon burning is a result, leaving mainly intermediate-mass-elements (IME) such as ${ }^{28} \mathrm{Si}$ and ${ }^{32} \mathrm{~S}$. At $T_{\max , 9} \sim 2-3$, carbon burning or neon burning is a result, characterized by abundant ${ }^{16} \mathrm{O}$ and ${ }^{24} \mathrm{Mg}$ in consumption of ${ }^{12} \mathrm{C}$. At even lower temperatures $\left(T_{\max , 9} \lesssim 2\right)$, virtually no major thermonuclear reactions take place.

The detonation wave can also be characterized by the same tendency, although the temperature reached in the ashes is not a unique function of the fuel density anymore but also depends on the shock strength, the effect of which needs further investigation in hydrodynamic simulations. In the delayed detonation framework, the detonation is ignited after significant expansion of the WD, and thus the electron captures are almost always unimportant (§3.4). If there is still a high density region left after the deflagration, the detonation wave can convert the material chiefly into ${ }^{56} \mathrm{Ni}$ by complete silicon burning. As the density decreases, the detonation nucleosynthesis is characterized by oxygen burning, carbon burning, and eventually burning ceases. Compared to the corresponding deflagration case at a similar burning stage, these stages are usually encountered at lower fuel densities, as the detonation compresses the burning material.

\subsection{Structure of the ejecta}

Table 1 shows the global features of our calculations. In the C-DEF model, more than half of the WD material is left unburned. The final kinetic energy, $\sim 4 \times 10^{50}$ erg, is significantly lower than that inferred for normal $\mathrm{SNe}$ Ia. In the $\mathrm{C}-\mathrm{DDT}$ model, $\sim 0.5 M_{\odot}$ of the $\mathrm{C} / \mathrm{O}$ material is incinerated by the detonation wave, after the deflagration already has burned $\sim 0.5 M_{\odot}$. The incinerated mass and nuclear energy release in the deflagration stage of the O-DDT model are smaller than the C-DDT model. The detonation wave, however, burns a larger amount of material and produces a larger amount of nuclear energy in the O-DDT model than in the C-DDT model. The final incinerated mass and the kinetic energy in the O-DDT model are comparable to those in the W7 model. Hereafter, we discuss how these results can be understood in terms of the flame propagation and the DDT. Note that the nuclear energy release in the hydrodynimic simulations (with a simplified treatment for the nuclear reactions) and that in the detailed reaction network calculations are consistent within $5 \%$.

Figures $1-3$ show the distribution of selected species at 10 seconds after the ignition of the deflagration flame. At this time, burning has ceased and the SN material is 

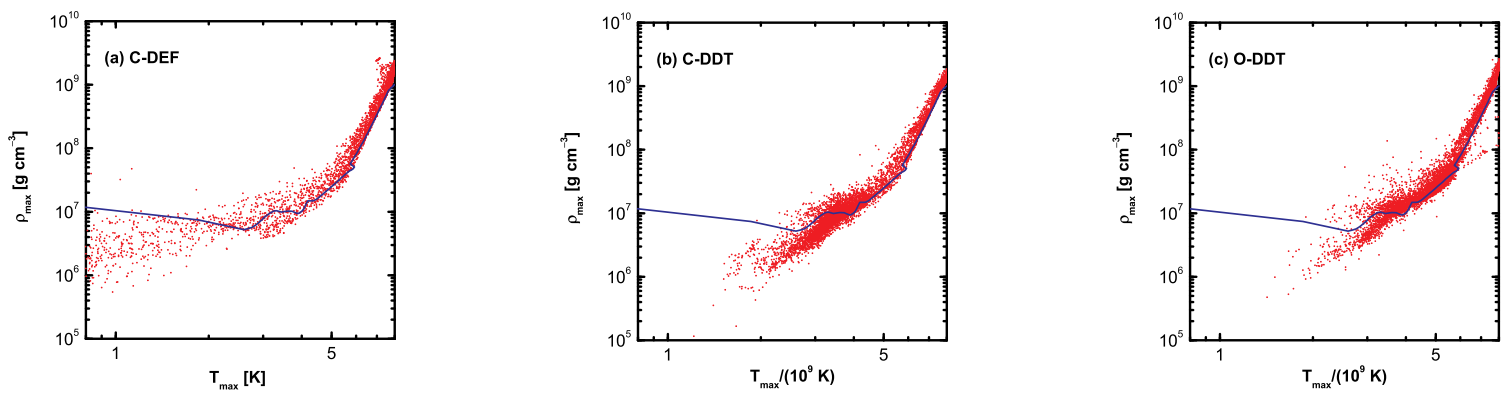

FIG. 6.- Maximum density $\rho_{\max }$ and temperature $T_{\max }$ for individual tracer particles (dots), after the passage of the thermonuclear flame. The solid line is for the W7 model.

already almost in a homologous expansion. The C-DEF model (Fig. 1) shows large-scale mixing, and thus it does not possess a clearly layered structure as predicted in exactly spherical 1D models (e.g., W7). A large amount of unburned carbon and oxygen are left, being mixed down to the central region.

In contrast, the detonation wave produces a more layered structure, since this supersonically propagating wave is unaffected by hydrodynamic instabilities (at least on the large scales resolved in the models). In the C-DDT model, the deflagration burns out much of the center of the WD before the detonation is triggered. Therefore, the detonation mainly burns material towards the stellar surface, but it also propagates down the fuel funnels between the fingers of the deflagration ash (Fig. 1). In the C-DDT model, the whole WD, including the central region, experiences the strong expansion in the vigorous deflagration stage. Therefore, the unburned material left after the deflagration is processed in the detonation stage mainly by carbon and oxygen burning, producing $\mathrm{O}$ and IME, but virtually no Fe-peak elements (Fig. 2). In this particular model, Fe-peak elements are thus produced mostly in the initial deflagration stage.

In the O-DDT model the burning proceeds in an aspherical manner. Figures 4 and 5 show the temporal evolution in the O-DDT model. The deflagration frame, ignited off-center, floats outward, and spreads laterally (Röpke et al. 2007d). It creates a large blob of ashes in the upper hemisphere of the WD. Due to the lateral expansion in the outer layers of the star, the neutron-rich Fe-peak elements produced by electron captures end up in a characteristic off-center shell-like layer (Fig. 3). The detonation triggers on top of this blob at $\sim 1$ second. It cannot cross ash regions (Maier \& Niemeyer 2006) and has to burn around the ash blob in order to reach the central parts of the WD. Consequently, it initially propagates only outward (at $\sim 1.1$ second).

At about $1.5 \mathrm{sec}$ after the deflagration ignition, the detonation wave has burned around the ash blob and propagates inward. Since the off-center deflagration did not release much energy, the central parts of the WD are still dense and contain mostly unburned material. This material is converted to Fe-peak (predominantly ${ }^{56} \mathrm{Ni}$ ) by the detonation wave.

\subsection{Characteristic thermal properties}

To understand the characteristic structure of the ejecta described in $§ 3.2$, we examine details of the thermal his-

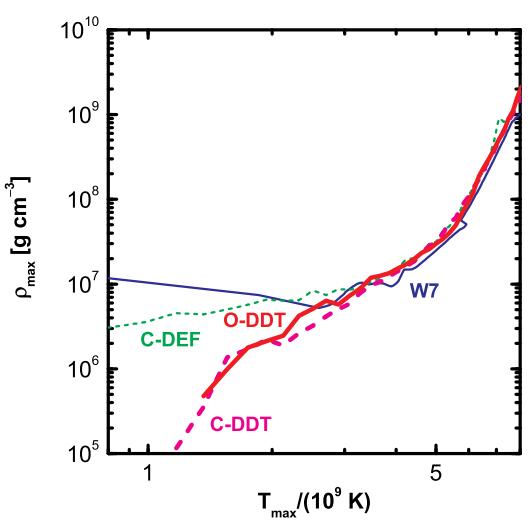

FIG. 7.- Average peak density of tracer particles as a function of peak temperature (with the temperature bin taken to be $\Delta T_{\max }=$ $2 \times 10^{8} \mathrm{~K}$ ). Models are W7 (blue thin-solid), C-DEF (green thindashed), C-DDT (magenta thick-dashed), and O-DDT (red thicksolid).

tory of the tracer particles. Figure 6 shows the maximum temperature $\left(T_{\max }\right)$ and density $\left(\rho_{\max }\right)$ for each tracer particle (§3.1) after flame passage. For comparison, the same values for the classical W7 model are also shown. In Figure 7, average behaviors are plotted, which were obtained by taking the mass-average as a function of $T_{\max }$.

Figures 6 and 7 show that the distribution of $\left(T_{\max }\right.$, $\rho_{\text {max }}$ ) is similar for all the models at $T_{\max , 9} \gtrsim 3$ (corresponding to $\left.\rho_{\max } \sim \rho_{\mathrm{DDT}}\right)$. This reflects the fact that the local properties of the deflagration flame are basically independent from the geometry. Interestingly, despite the different prescription of the deflagration flame propagation and different expansion time scale (see e.g., Travaglio et al. 2004a), we note a similarity with the outcome of the W7 in this respect.

At $T_{\max , 9} \lesssim 3$, the models differ. Note that the behavior of W7 in this temperature regime shown in Figures 6 and 7 is not real; the deflagration is turned off at $T_{\max , 9} \sim 2$, where the flame is already near the surface in the W7 model. Our deflagration model extends to lower $T_{\max }$ and $\rho_{\max }$. In the two delayed detonation models, this temperature range corresponds to the detonation phase, and thus the behavior is expected to deviate from the deflagration models, and indeed it does. The two detonation models show a similar $T_{\max }-\rho_{\max }$ distri- 


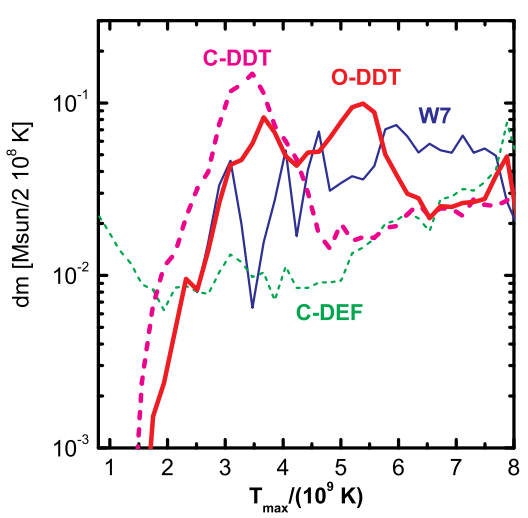

FIG. 8.- The masses $\left(\mathrm{M}_{\odot}\right)$ within each peak temperature range $\left(\Delta T=2 \times 10^{8} \mathrm{~K}\right)$. Models are W7 (blue thin-solid), C-DEF (green thin-dashed), C-DDT (magenta thick-dashed), and O-DDT (red thick-solid).

bution; the compression and the maximum temperature are roughly linear functions of the unburned density.

Figure 6 shows a significant dispersion in the $T_{\max }-$ $\rho_{\max }$ distribution around the mean value, reaching nearly one order of magnitude in $\rho_{\max }$ for given $T_{\max }$, at $T_{\max , 9} \lesssim 5$. This is a unique behavior in the multi-D models, potentially leading to a variety of burning products otherwise not expected in strictly spherically symmetric models like W7.

The different hydrodynamic properties lead to different amounts of material as a function of $T_{\max }\left(\right.$ or $\left.\rho_{\max }\right)$, and this is the main reason why different nucleosynthesis features appear. Figure 8 shows the mass of the material as a function of $T_{\max }$. It is seen that the deflagration is efficient in W7, and thus the W7 model has a much larger amount of the mass with $T_{\max , 9} \gtrsim 6$ than the $2 \mathrm{D}$ models. At $T_{\max , 9} \lesssim 3-4$, there appears a peak in the C-DDT model; this is due to the detonation wave burning the fuel material left behind the deflagration stage. The O-DDT model shows a characteristic distribution; two peaks at $T_{\max , 9} \sim 3-4$ and at $T_{\max , 9} \sim 5-6$. The former is the detonation wave propagating outward, as also seen in the C-DDT model. The latter one at higher $T_{\max }$ is due to the detonation wave propagating inward, near the central region where the fuel still has high densities. The gap between the two peaks corresponds to the (off-center) shell structure of the deflagration products (i.e., neutron-rich Fe-peak elements; Fig. 3).

\subsection{Deflagration and Electron Captures}

In the densest region in the deflagration stage, electron capture reactions proceed to synthesize neutron-rich Fepeak elements under NSE conditions. Figure 9 shows the electron fraction $\left(Y_{\mathrm{e}}\right)$ as a function of $\rho_{\max }$, for the O-DDT model. The same figure for the other 2D models (not shown) is similar to the O-DDT model. Figure 9 shows that the electron capture becomes important at $\rho_{\max } \gtrsim 5 \times 10^{8} \mathrm{~g} \mathrm{~cm}^{-3}$, and $Y_{\mathrm{e}}$ as low as 0.463 is realized in the highest density region. Thus, the main product of the highest density region is stable ${ }^{56} \mathrm{Fe}\left(Y_{\mathrm{e}} \sim 0.464\right)$, followed by ${ }^{54} \mathrm{Fe}\left(Y_{\mathrm{e}} \sim 0.481\right)$ and ${ }^{58} \mathrm{Ni}\left(Y_{\mathrm{e}} \sim 0.483\right)$ in the lower density region. Since $Y_{\mathrm{e}}>0.46,{ }^{50} \mathrm{Ti}\left(Y_{\mathrm{e}} \sim 0.440\right)$, ${ }^{54} \mathrm{Cr}\left(Y_{\mathrm{e}} \sim 0.444\right)$ and ${ }^{58} \mathrm{Fe}\left(Y_{\mathrm{e}} \sim 0.448\right)$ are not pro-

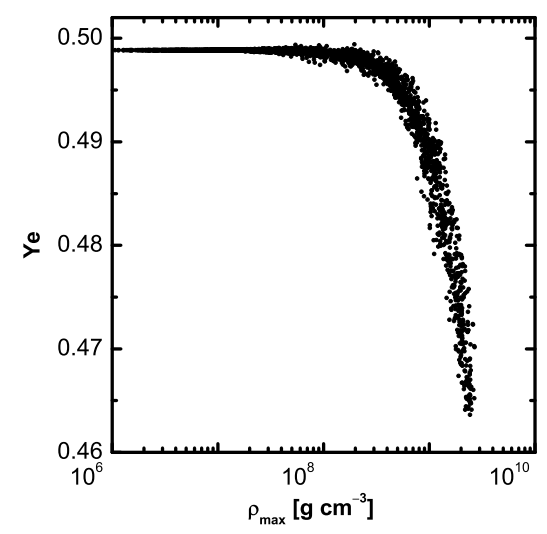

FIG. 9.- The final values of $Y_{\mathrm{e}}$ as a function of the peak density, for the O-DDT model.

duced abundantly in the present models. The behavior in $Y_{\mathrm{e}}$ and the resulting electron capture reactions are largely consistent with the 2D deflagration model presented in Travaglio et al. (2004a). The electron captures, however, are less efficient in the 2D models than in the 3D deflagration model (Travaglio et al. 2004a) and in the classical 1D models (Thielemann et al. 2004; see also §3.6). This is likely due to the weaker deflagration in the present models than in the others (1D and 3D), although an uncertainty is involved in the treatment of the temperature evolution in the NSE regime (§2.2).

Figure 10 shows the comparison between $Y_{\mathrm{e}}$ before and after the DDT. In the C-DDT model, $Y_{\mathrm{e}}$ is not affected by the detonation wave as is consistent with previous $1 \mathrm{D}$ models. On the other hand, in the O-DDT model the detonation slightly affects $Y_{\mathrm{e}}$ especially of the material which was hardly processed by the deflagration $\left(\sim Y_{\mathrm{e}} \gtrsim\right.$ 0.496). The change in $Y_{\mathrm{e}}$ introduced by the detonation is at most $\Delta Y_{\mathrm{e}} \sim 0.02$, much smaller than the change in the deflagration stage at $\gtrsim 10^{8}-10^{9} \mathrm{~g} \mathrm{~cm}^{-3}$. This is a result of electron capture reactions at the detonation wave propagating near the WD center, since the central region is still at high density (e.g., Meakin et al. 2009). The change is not as large as that in the GCD models of Jordan et al. (2008) and Meakin et al. (2009), which resulted in $\Delta Y_{\mathrm{e}} \sim 0.05$. This is because the distribution of the initial bubbles in our simulations is not as extreme as theirs (see \$2.1) and the WD pre-expansion before the onset of the detonation was stronger in our model.

\subsection{Distribution of Nucleosynthesis Products}

Figure 11 shows the radial abundance distribution, for which the material is angle-averaged within each velocity bin. Note that our models are followed up to $\sim 10$ seconds, thus the velocity is proportional to the radial distance, and the distribution in velocity space corresponds in good approximation to that in spatial space. The O-DDT model has a very aspherical ejecta structure. Therefore, the angle-dependent radial abundance distribution in this model is given in Figure 12 .

In the C-DEF model, different burning regions are macroscopically well-mixed. As a consequence, it forms a broad region in which the electron capture products $\left({ }^{56} \mathrm{Fe},{ }^{54} \mathrm{Fe}\right.$, and $\left.{ }^{58} \mathrm{Ni}\right)$, complete-silicon burning products 

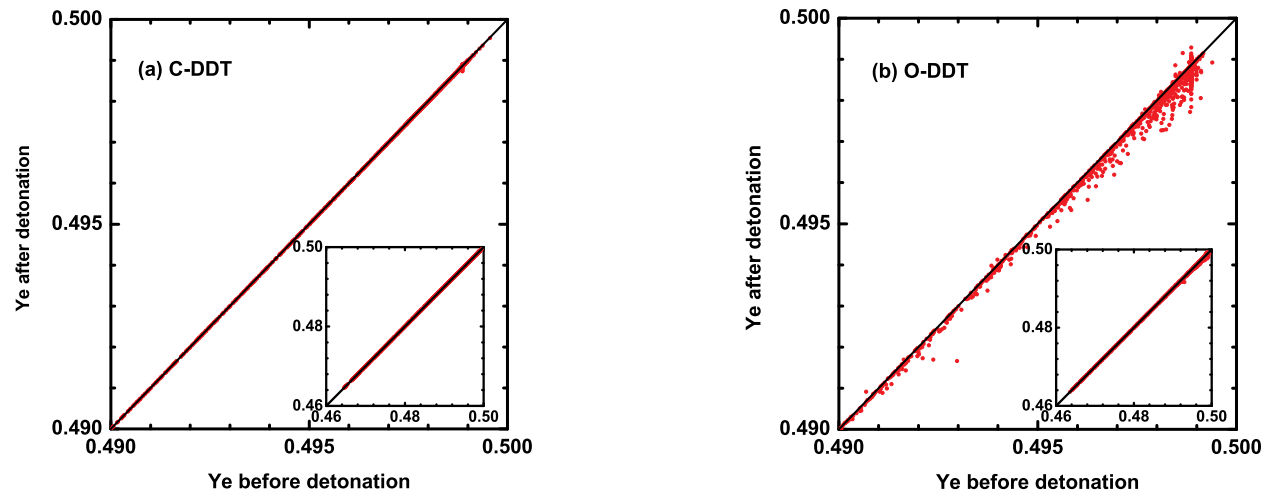

FIG. 10.- The final values of $Y_{\mathrm{e}}$ (i.e., after the detonation) as compared to those just before the first DDT transition (i.e., before the detonation), for (a) C-DDT and (b) O-DDT models (shown by dots).

$\left({ }^{56} \mathrm{Ni}\right)$, intermediate mass elements $\left({ }^{32} \mathrm{~S},{ }^{28} \mathrm{Si},{ }^{24} \mathrm{Mg}\right)$, and unburned elements $\left({ }^{16} \mathrm{O}\right.$ and ${ }^{12} \mathrm{C}$ ) coexist (when averaging in the zenith angle). This region is surrounded by unburned material at radii not reached by the flame. An enhancement of $\mathrm{C}$ and $\mathrm{O}$ near the center due to the largescale deflagration mixing is visible. These are typical of $2 \mathrm{D}$ deflagration models; this smoothing effect tends to be suppressed in 3D deflagration models (Röpke et al. 2007b).

In the C-DDT model, the inner region below $\sim 8,000$ $\mathrm{km} \mathrm{s}^{-1}$ has a similar structure to that in the deflagration model. The difference is seen in the innermost region, where unburned $\mathrm{C}$ and $\mathrm{O}$ are burned into intermediate mass elements by the detonation wave. In this model, $\mathrm{C}$ is almost completely burned, but there still remains $\mathrm{O}$ with a mass fraction of $\sim 0.1$, since the detonation in this model leads to carbon or oxygen burning.

Surrounding the deflagration region is the oxygen burning layer, where ${ }^{28} \mathrm{Si},{ }^{32} \mathrm{~S}$, and ${ }^{24} \mathrm{Mg}$ are the main products. This is surrounded by a mixture of carbon burning products and unburned material. Note that the carbon burning region and the unburned region are microscopically separated. As shown in Figure 2, the unburned region (with almost initial $\mathrm{C}+\mathrm{O}$ composition) is not fully homogeneously distributed, and there are "fingers" of carbon burning regions (where oxygen and IMEs are produced in consumption of carbon).

The radial composition distribution in the O-DDT model follows that of the C-DDT model. A striking difference, however, is that the region of deflagration ashes is not in the center but confined within $V \sim$ $5,000-10,000 \mathrm{~km} \mathrm{~s}^{-1}$. The innermost region is converted to ${ }^{56} \mathrm{Ni}$, by the complete silicon burning taking place at the inward detonation wave.

\subsection{Total Yields}

Figure 13 shows the nucleosynthesis yield for each model, integrated over the whole ejecta. The ratios of the masses of elements to those of the W7 model are plotted in Figure 14. Tables 2 and 3 list the masses of the elements and isotopes (after radioactive decays) while major long-lived radioactive isotopes are given in Table 4. For the C-DDT and O-DDT models, the values at the time of the first DDT $(\sim 1.15 \mathrm{sec}$ for $\mathrm{C}-\mathrm{DDT}$ and $\sim 1.05$ sec for O-DDT) are also shown in Tables 2 and 3.
For comparison, we present the yields of the W7 model, calculated by ourselves using the thermal history of the original model. Details are different from Iwamoto et al. (1999), because of the updated electron capture rates (see Brachwitz et al. 2000).

The result for the C-DEF model is consistent with the similar 2D model in the previous study (Travaglio et al. 2004a). First of all, the mass of ${ }^{56} \mathrm{Ni}$ is only $\sim 0.25 M_{\odot}$, smaller than in the $\mathrm{W} 7$ model $\left(\sim 0.64 M_{\odot}\right)$. A large amount of $\mathrm{C}$ and $\mathrm{O}$ are left unburned. The final $\mathrm{C} / \mathrm{O}$ ratio is larger than in the W7 model, because of the weak C-burning in our model resulting in almost the original, unburned $\mathrm{C} / \mathrm{O}$ ratio near the surface (Fig. 11). IMEs are underproduced as compared to the W7 model. Note, however, that generally full three-dimensional models alleviate this problem (e.g., Travaglio et al. 2004a; Röpke et al. 2007b).

In the C-DDT model, the density of the WD is already low when the DDT takes place, and the temperature of material after the passage of the detonation wave does not reach $\sim 5 \times 10^{9} \mathrm{~K}$ (Fig. 8). The detonation therefore produces at most IMEs; Fe-peak elements are produced in the initial deflagration stage, and the mass of ${ }^{56} \mathrm{Ni}$ is almost the same as in the deflagration model. Consequently, the abundance pattern of the Fe-peak elements (including ${ }^{56} \mathrm{Ni}$ ) is very similar to that of the C-DEF model (Figs. 13 and 14). IMEs are produced much more abundantly than in the W7 model. Because of the carbon burning, the $\mathrm{C} / \mathrm{O}$ ratio is much smaller than for $\mathrm{W} 7$.

Somewhat surprisingly, the O-DDT model predicts the abundance pattern similar to W7 (Fig. 13), with most of elements consistent with W7 within a factor of two (Fig. 14). The mass of ${ }^{56} \mathrm{Ni}\left(\sim 0.54 M_{\odot}\right)$ is comparable with the W7 model $\left(\sim 0.64 M_{\odot}\right)$ (but see $\left.\S 4\right)$. IMEs tend to be overproduced by a factor of $\sim 2$, because of the carbon and oxygen burning in the detonation phase. Carbon is almost entirely consumed by the carbon burning.

A comparison between the W7 model and our 2D models shows that very strong electron capture reactions are not efficient in the $2 \mathrm{D}$ models $(\S 3.4) .{ }^{50} \mathrm{Ti},{ }^{54} \mathrm{Cr}$, and ${ }^{58} \mathrm{Fe}$, produced at the highest density resulting in $Y_{\mathrm{e}}<0.46$, are not abundantly produced in the $2 \mathrm{D}$ models. 

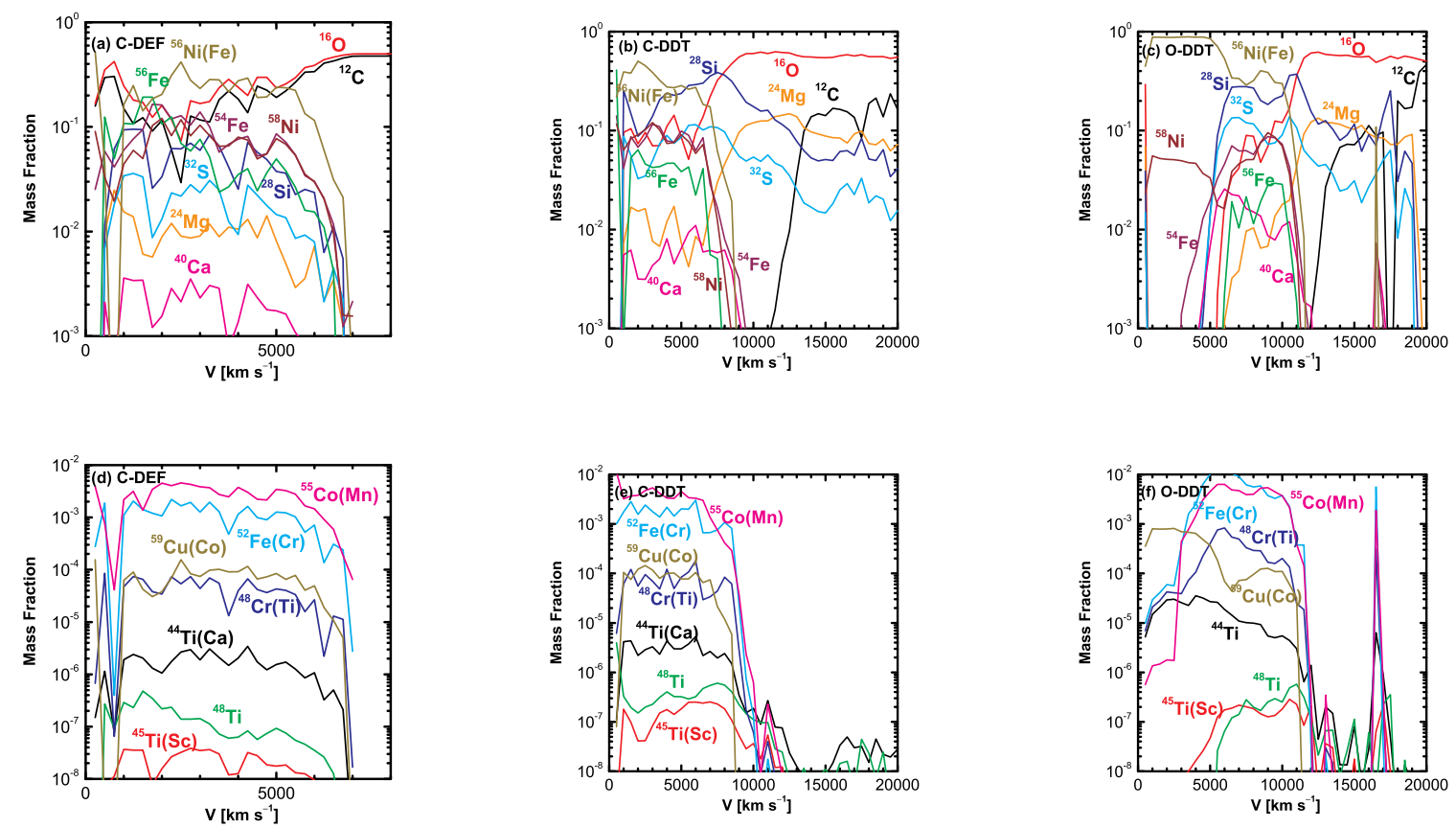

FIG. 11. - Mass fractions of selected isotopes as a function of the expansion velocity, averaged in the velocity bin of $\Delta V$. (a, d) C-DEF model, $\Delta V=250 \mathrm{~km} \mathrm{~s}^{-1}$, (b, e) C-DDT, $\Delta V=500 \mathrm{~km} \mathrm{~s}^{-1}$, and (c, f) O-DDT, $\Delta V=500 \mathrm{~km} \mathrm{~s}^{-1}$.
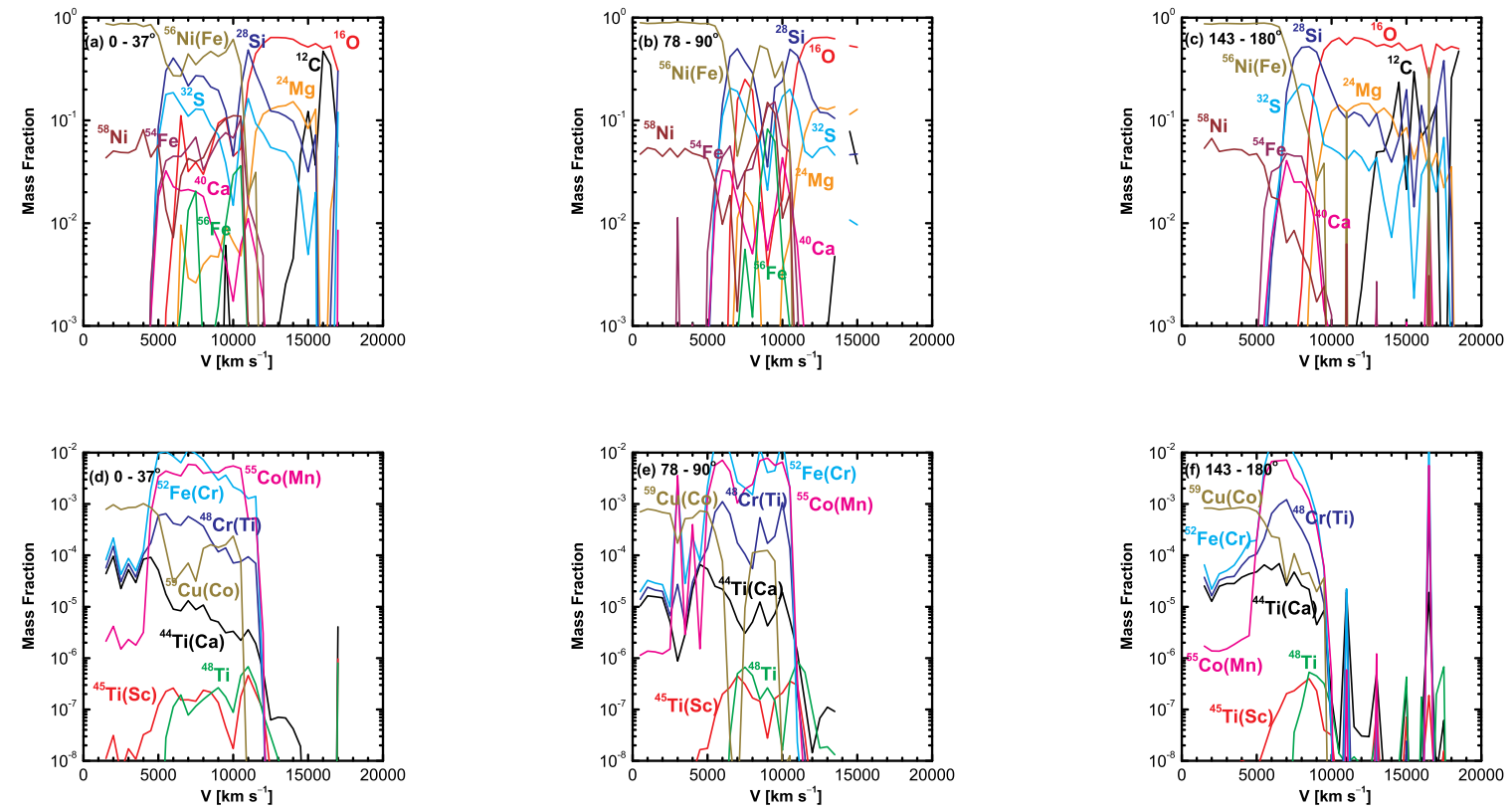

FIG. 12.- Angle-dependent mass fractions of selected isotopes in O-DDT, as a function of the expansion velocity, averaged in the velocity bin of $\Delta V=500 \mathrm{~km} \mathrm{~s}^{-1}$. (a, d) averaged within $0-37 \mathrm{deg}$, (b, e) $78-90 \mathrm{deg}$, and (c, f) $143-180 \mathrm{deg}$. 

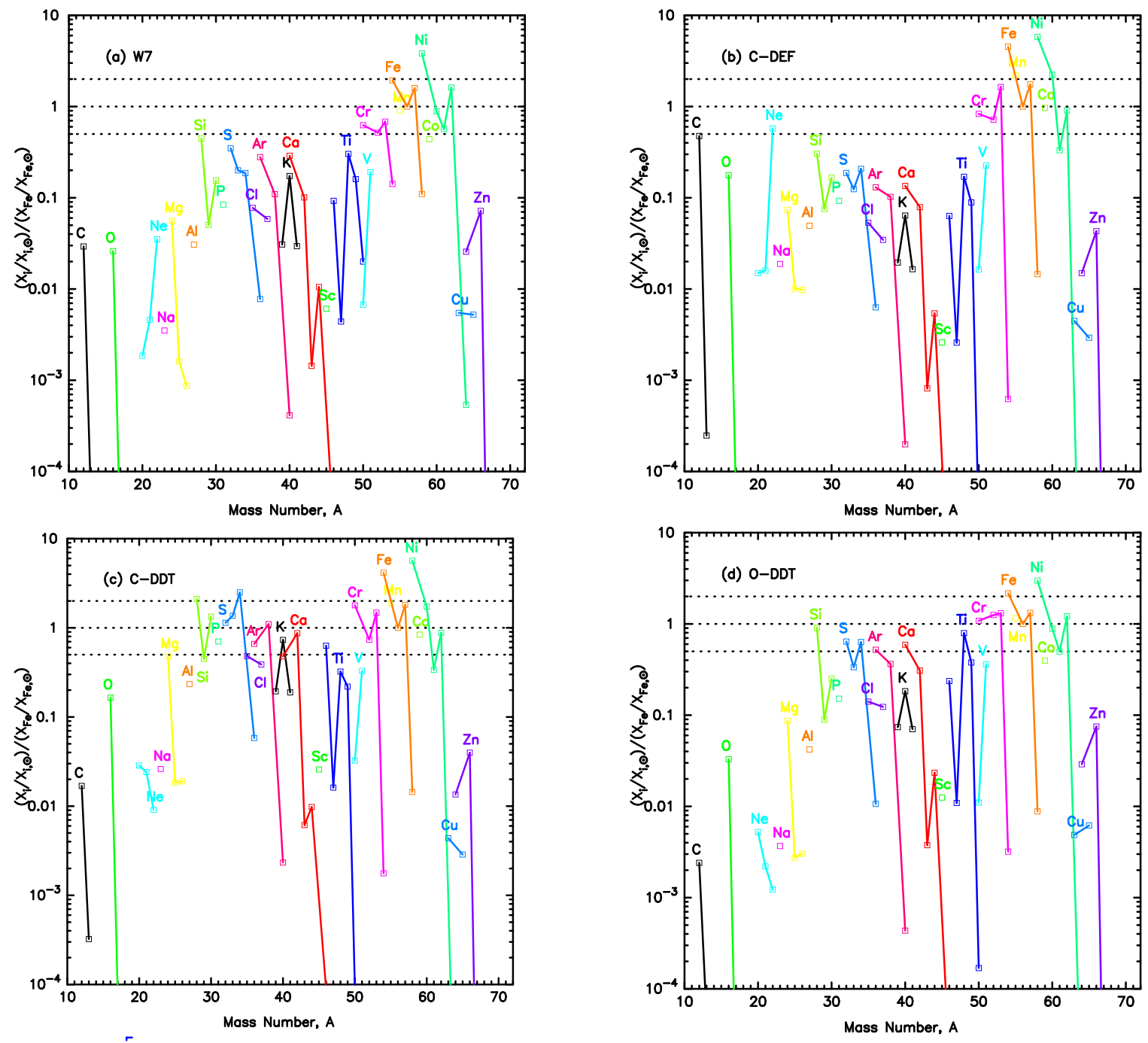

FIG. 13.- Isotopic yields integrated within the whole ejecta (after radioactive decays). The mass fraction is shown relative to the solar value, and normalized by ${ }^{56} \mathrm{Fe}$.
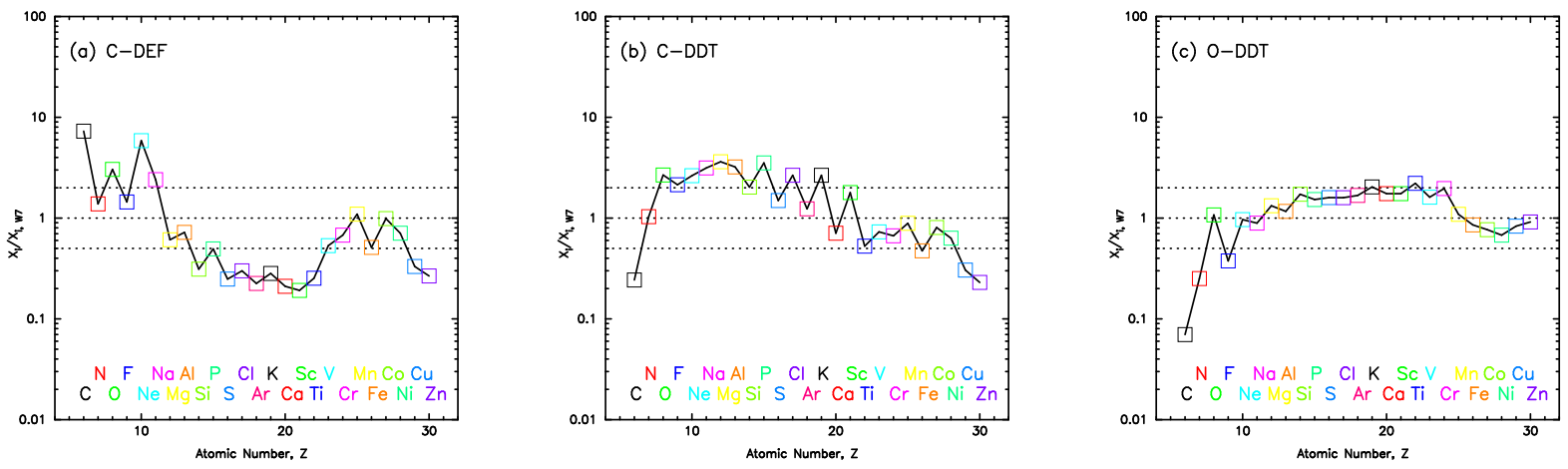

FIG. 14.- Ratios of integrated element yields to the W7 model yields. 
TABLE 2

MAsses of ELEMENTS $\left(\mathrm{M}_{\odot}\right)$

\begin{tabular}{ccccccc}
\hline \hline Element & W7 & C-DEF & C-DDT & O-DDT & C-DDT/def. & O-DDT/def. \\
\hline $\mathrm{C}$ & $4.99 \mathrm{E}-02$ & $3.64 \mathrm{E}-01$ & $1.21 \mathrm{E}-02$ & $3.49 \mathrm{E}-03$ & $4.17 \mathrm{E}-01$ & $4.86 \mathrm{E}-01$ \\
$\mathrm{~N}$ & $1.11 \mathrm{E}-06$ & $1.54 \mathrm{E}-06$ & $1.15 \mathrm{E}-06$ & $2.80 \mathrm{E}-07$ & $2.55 \mathrm{E}-06$ & $1.14 \mathrm{E}-05$ \\
$\mathrm{O}$ & $1.40 \mathrm{E}-01$ & $4.27 \mathrm{E}-01$ & $3.76 \mathrm{E}-01$ & $1.51 \mathrm{E}-01$ & $4.61 \mathrm{E}-01$ & $5.21 \mathrm{E}-01$ \\
$\mathrm{~F}$ & $7.19 \mathrm{E}-10$ & $1.04 \mathrm{E}-09$ & $1.54 \mathrm{E}-09$ & $2.70 \mathrm{E}-10$ & $5.40 \mathrm{E}-10$ & $3.56 \mathrm{E}-10$ \\
$\mathrm{Ne}$ & $4.28 \mathrm{E}-03$ & $2.51 \mathrm{E}-02$ & $1.13 \mathrm{E}-02$ & $4.13 \mathrm{E}-03$ & $2.49 \mathrm{E}-02$ & $2.82 \mathrm{E}-02$ \\
$\mathrm{Na}$ & $6.59 \mathrm{E}-05$ & $1.59 \mathrm{E}-04$ & $2.07 \mathrm{E}-04$ & $5.87 \mathrm{E}-05$ & $7.09 \mathrm{E}-05$ & $6.15 \mathrm{E}-05$ \\
$\mathrm{Mg}$ & $1.63 \mathrm{E}-02$ & $9.92 \mathrm{E}-03$ & $5.90 \mathrm{E}-02$ & $2.16 \mathrm{E}-02$ & $4.81 \mathrm{E}-03$ & $2.27 \mathrm{E}-03$ \\
$\mathrm{Al}$ & $1.00 \mathrm{E}-03$ & $7.23 \mathrm{E}-04$ & $3.22 \mathrm{E}-03$ & $1.17 \mathrm{E}-03$ & $3.45 \mathrm{E}-04$ & $1.99 \mathrm{E}-04$ \\
$\mathrm{Si}$ & $1.67 \mathrm{E}-01$ & $5.19 \mathrm{E}-02$ & $3.38 \mathrm{E}-01$ & $2.87 \mathrm{E}-01$ & $3.39 \mathrm{E}-02$ & $1.38 \mathrm{E}-02$ \\
$\mathrm{P}$ & $3.86 \mathrm{E}-04$ & $1.91 \mathrm{E}-04$ & $1.36 \mathrm{E}-03$ & $5.91 \mathrm{E}-04$ & $1.34 \mathrm{E}-04$ & $8.34 \mathrm{E}-05$ \\
$\mathrm{~S}$ & $7.97 \mathrm{E}-02$ & $1.98 \mathrm{E}-02$ & $1.19 \mathrm{E}-01$ & $1.27 \mathrm{E}-01$ & $1.33 \mathrm{E}-02$ & $6.05 \mathrm{E}-03$ \\
$\mathrm{Cl}$ & $1.38 \mathrm{E}-04$ & $4.15 \mathrm{E}-05$ & $3.69 \mathrm{E}-04$ & $2.20 \mathrm{E}-04$ & $5.81 \mathrm{E}-05$ & $7.83 \mathrm{E}-05$ \\
$\mathrm{Ar}$ & $1.31 \mathrm{E}-02$ & $2.94 \mathrm{E}-03$ & $1.62 \mathrm{E}-02$ & $2.20 \mathrm{E}-02$ & $2.41 \mathrm{E}-03$ & $1.61 \mathrm{E}-03$ \\
$\mathrm{~K}$ & $6.45 \mathrm{E}-05$ & $1.82 \mathrm{E}-05$ & $1.71 \mathrm{E}-04$ & $1.31 \mathrm{E}-04$ & $3.25 \mathrm{E}-05$ & $8.11 \mathrm{E}-05$ \\
$\mathrm{Ca}$ & $9.76 \mathrm{E}-03$ & $2.05 \mathrm{E}-03$ & $6.91 \mathrm{E}-03$ & $1.70 \mathrm{E}-02$ & $1.72 \mathrm{E}-03$ & $1.60 \mathrm{E}-03$ \\
$\mathrm{Sc}$ & $1.33 \mathrm{E}-07$ & $2.55 \mathrm{E}-08$ & $2.38 \mathrm{E}-07$ & $2.29 \mathrm{E}-07$ & $1.59 \mathrm{E}-07$ & $1.59 \mathrm{E}-05$ \\
$\mathrm{Ti}$ & $3.94 \mathrm{E}-04$ & $9.97 \mathrm{E}-05$ & $2.08 \mathrm{E}-04$ & $8.72 \mathrm{E}-04$ & $1.53 \mathrm{E}-04$ & $1.98 \mathrm{E}-03$ \\
$\mathrm{~V}$ & $4.04 \mathrm{E}-05$ & $2.15 \mathrm{E}-05$ & $2.95 \mathrm{E}-05$ & $6.52 \mathrm{E}-05$ & $1.29 \mathrm{E}-04$ & $3.01 \mathrm{E}-03$ \\
$\mathrm{Cr}$ & $5.28 \mathrm{E}-03$ & $3.57 \mathrm{E}-03$ & $3.52 \mathrm{E}-03$ & $1.04 \mathrm{E}-02$ & $4.41 \mathrm{E}-03$ & $2.07 \mathrm{E}-02$ \\
$\mathrm{Mn}$ & $6.72 \mathrm{E}-03$ & $7.35 \mathrm{E}-03$ & $5.95 \mathrm{E}-03$ & $7.35 \mathrm{E}-03$ & $1.83 \mathrm{E}-02$ & $4.42 \mathrm{E}-02$ \\
$\mathrm{Fe}$ & $7.61 \mathrm{E}-01$ & $3.89 \mathrm{E}-01$ & $3.60 \mathrm{E}-01$ & $6.51 \mathrm{E}-01$ & $3.36 \mathrm{E}-01$ & $2.06 \mathrm{E}-01$ \\
$\mathrm{Co}$ & $8.29 \mathrm{E}-04$ & $8.20 \mathrm{E}-04$ & $6.70 \mathrm{E}-04$ & $6.36 \mathrm{E}-04$ & $1.31 \mathrm{E}-03$ & $3.22 \mathrm{E}-03$ \\
$\mathrm{Ni}$ & $1.19 \mathrm{E}-01$ & $8.42 \mathrm{E}-02$ & $7.50 \mathrm{E}-02$ & $8.05 \mathrm{E}-02$ & $6.88 \mathrm{E}-02$ & $4.30 \mathrm{E}-02$ \\
$\mathrm{Cu}$ & $2.55 \mathrm{E}-06$ & $8.45 \mathrm{E}-07$ & $7.82 \mathrm{E}-07$ & $2.13 \mathrm{E}-06$ & $1.38 \mathrm{E}-06$ & $1.26 \mathrm{E}-05$ \\
$\mathrm{Zn}$ & $3.80 \mathrm{E}-05$ & $1.01 \mathrm{E}-05$ & $8.82 \mathrm{E}-06$ & $3.50 \mathrm{E}-05$ & $1.08 \mathrm{E}-06$ & $5.48 \mathrm{E}-06$ \\
& & & & & & \\
\hline $\mathrm{At}$ & & & & & \\
\hline
\end{tabular}

${ }^{\mathrm{a}}$ At the moment when the first DDT takes place.

\subsection{Radioactive Isotopes}

The amount of some radioactive isotopes in our detonation models is quite different from W7 and even with canonical, spherical delayed detonation models. Comparison between our results (Tab. 4) and Table 4 of Iwamoto et al. (1999) shows that radioactive isotopes lighter than Fe-peak tend to be more abundant, with the tendency of larger amounts of these isotopes for smaller density at the detonation. On the other hand, the amount of some Fe-peak radioactive isotopes can change dramatically from the classical 1D delayed detonation model, due to updated electron capture rates in our calculations $(\S 2.2)$.

\section{DISCUSSION: IMPLICATIONS FOR CHEMICAL EVOLUTION}

In this paper, we present our first results for nucleosynthesis in 2D delayed detonation models. The models investigated in this paper represent extreme cases ${ }^{6}$; the deflagration is initiated either at the center (C-DDT) or by off-center bubbles confined in the narrow angle with respect to the $z$-axis (O-DDT). The important variation, we have not examined in this paper, is the deflagration bubbles distributed more or less spherically, but at a distance from the center (e.g., Kasen et al. 2009). In this case, we expect that the propagation of the burning will produce an ejecta structure that falls in between the $\mathrm{O}$ DDT and the C-DDT models. Some material in the central region is left unexpanded in the deflagration stage, and the detonation can burn the material there to the Fe-peak elements. Thus, we expect that nucleosynthesis features are similar to the O-DDT model, except for the strong angle-dependence seen in the O-DDT model.

\footnotetext{
6 Note that the O-DDT model is still less extreme than the GCD model (Jordan et al. 2008; Meakin et al. 2009) in the distribution of the initial bubbles.
}

Depending on the initial placement of the deflagration bubbles, the deflagration ashes may stay more confined to the center than in the O-DDT model.

Also, we should note that the treatment of the DDT in the hydrodynamic calculation is still preliminary ( $\$ 2.1)$. According to simulations with several different prescriptions, especially with different values for $\rho_{\mathrm{DDT}}$, we believe that the prescription used in this paper results in a relatively weak detonation (see also Gamezo et al. 2005; Bravo \& García-Senz 2008). Therefore, although the models presented here are extreme with respect to the ejecta structure, they do not necessarily reflect the range of ${ }^{56} \mathrm{Ni}$ production possible within the framework of the delayed detonation model. The results of an extended survey of delayed detonation models will be presented elsewhere (F. Röpke et al., in prep). Despite these caveats, the general features found in this paper are not expected to be sensitive to such details.

The nucleosynthesis features in the W7 model have been shown to be roughly consistent with the Galactic chemical evolution (e.g., Iwamoto et al. 1999; Goswami \& Prantzos 2000). One problem is that the ratio ${ }^{58} \mathrm{Ni} /{ }^{56} \mathrm{Fe}$ is too large in the W7 to compensate the over-solar production of ${ }^{58} \mathrm{Ni}$ in core-collapse SNe. The problem is basically solved by introducing a delayed detonation. Affecting primarily lower density material, it produces a large amount of ${ }^{56} \mathrm{Ni}$ but virtually no ${ }^{58} \mathrm{Ni}$ due to the inefficiency of electron captures here. We see that the ratio ${ }^{58} \mathrm{Ni} /{ }^{56} \mathrm{Fe}$ is slightly decreased in the O-DDT model compared to the W7, marginally satisfying the constraint from the chemical evolution $\left[\left({ }^{58} \mathrm{Ni} /{ }^{56} \mathrm{Fe}\right) /\left({ }^{58} \mathrm{Ni} /{ }^{56} \mathrm{Fe}\right) \odot\right.$ $\sim 3]$. The situation should become better if the detonation burns more material to NSE than in the present prescription. We should also note one limitation in the present analysis, i.e., the treatment of the temperature in the NSE regime. The temperature is extracted assuming 
TABLE 3

MASSES OF ISOTOPES $\left(\mathrm{M}_{\odot}\right)$

\begin{tabular}{|c|c|c|c|c|c|c|}
\hline Isotopes & W7 & C-DEF & C-DDT & O-DDT & C-DDT / def. & O-DDT / def. \\
\hline${ }^{12} \mathrm{C}$ & $4.99 \mathrm{E}-02$ & $3.64 \mathrm{E}-01$ & $1.21 \mathrm{E}-02$ & $3.49 \mathrm{E}-03$ & $4.17 \mathrm{E}-01$ & $4.86 \mathrm{E}-01$ \\
\hline${ }^{13} \mathrm{C}$ & $9.57 \mathrm{E}-07$ & $2.29 \mathrm{E}-06$ & $2.80 \mathrm{E}-06$ & $8.75 \mathrm{E}-07$ & $1.16 \mathrm{E}-06$ & $1.35 \mathrm{E}-06$ \\
\hline${ }^{14} \mathrm{~N}$ & $1.11 \mathrm{E}-06$ & $1.54 \mathrm{E}-06$ & $1.15 \mathrm{E}-06$ & $2.79 \mathrm{E}-07$ & $2.55 \mathrm{E}-06$ & $1.14 \mathrm{E}-05$ \\
\hline${ }^{15} \mathrm{~N}$ & $1.71 \mathrm{E}-09$ & 1.65E-09 & 3.32E-09 & $7.06 \mathrm{E}-10$ & 2.79E-09 & $9.71 \mathrm{E}-09$ \\
\hline${ }^{16} \mathrm{O}$ & $1.40 \mathrm{E}-01$ & $4.27 \mathrm{E}-01$ & $3.76 \mathrm{E}-01$ & $1.51 \mathrm{E}-01$ & $4.62 \mathrm{E}-01$ & $5.21 \mathrm{E}-01$ \\
\hline${ }^{17} \mathrm{O}$ & $3.59 \mathrm{E}-08$ & $4.51 \mathrm{E}-08$ & $7.61 \mathrm{E}-08$ & $1.46 \mathrm{E}-08$ & $7.60 \mathrm{E}-08$ & $3.96 \mathrm{E}-07$ \\
\hline${ }^{18} \mathrm{O}$ & $1.13 \mathrm{E}-09$ & $1.80 \mathrm{E}-09$ & $2.42 \mathrm{E}-09$ & $5.24 \mathrm{E}-10$ & $2.04 \mathrm{E}-09$ & $2.57 \mathrm{E}-08$ \\
\hline${ }^{19} \mathrm{~F}$ & $7.19 \mathrm{E}-10$ & $1.04 \mathrm{E}-09$ & $1.54 \mathrm{E}-09$ & $2.70 \mathrm{E}-10$ & $5.40 \mathrm{E}-10$ & $3.56 \mathrm{E}-10$ \\
\hline${ }^{20} \mathrm{Ne}$ & $1.69 \mathrm{E}-03$ & $6.13 \mathrm{E}-03$ & $1.10 \mathrm{E}-02$ & $4.05 \mathrm{E}-03$ & $3.06 \mathrm{E}-03$ & $2.65 \mathrm{E}-03$ \\
\hline${ }^{21} \mathrm{Ne}$ & $1.06 \mathrm{E}-05$ & $1.66 \mathrm{E}-05$ & $2.36 \mathrm{E}-05$ & $4.35 \mathrm{E}-06$ & $7.40 \mathrm{E}-06$ & $4.09 \mathrm{E}-06$ \\
\hline${ }^{22} \mathrm{Ne}$ & $2.57 \mathrm{E}-03$ & $1.90 \mathrm{E}-02$ & $2.82 \mathrm{E}-04$ & $7.62 \mathrm{E}-05$ & $2.18 \mathrm{E}-02$ & $2.55 \mathrm{E}-02$ \\
\hline${ }^{23} \mathrm{Na}$ & $6.59 \mathrm{E}-05$ & $1.59 \mathrm{E}-04$ & $2.07 \mathrm{E}-04$ & $5.87 \mathrm{E}-05$ & $7.09 \mathrm{E}-05$ & $6.15 \mathrm{E}-05$ \\
\hline${ }^{24} \mathrm{Mg}$ & $1.62 \mathrm{E}-02$ & $9.55 \mathrm{E}-03$ & $5.84 \mathrm{E}-02$ & $2.14 \mathrm{E}-02$ & $4.61 \mathrm{E}-03$ & $2.14 \mathrm{E}-03$ \\
\hline${ }^{25} \mathrm{Mg}$ & $6.12 \mathrm{E}-05$ & $1.72 \mathrm{E}-04$ & $2.95 \mathrm{E}-04$ & $8.90 \mathrm{E}-05$ & $9.50 \mathrm{E}-05$ & $6.84 \mathrm{E}-05$ \\
\hline${ }^{26} \mathrm{Mg}$ & 3.79E-05 & $1.93 \mathrm{E}-04$ & $3.49 \mathrm{E}-04$ & $1.13 \mathrm{E}-04$ & $1.12 \mathrm{E}-04$ & $5.94 \mathrm{E}-05$ \\
\hline $27 \mathrm{Al}$ & $1.00 \mathrm{E}-03$ & $7.23 \mathrm{E}-04$ & $3.22 \mathrm{E}-03$ & $1.17 \mathrm{E}-03$ & $3.45 \mathrm{E}-04$ & $1.99 \mathrm{E}-04$ \\
\hline${ }^{28} \mathrm{Si}$ & $1.64 \mathrm{E}-01$ & $5.02 \mathrm{E}-02$ & $3.27 \mathrm{E}-01$ & $2.83 \mathrm{E}-01$ & $3.31 \mathrm{E}-02$ & $1.34 \mathrm{E}-02$ \\
\hline${ }^{29} \mathrm{Si}$ & $9.72 \mathrm{E}-04$ & $6.52 \mathrm{E}-04$ & $3.64 \mathrm{E}-03$ & $1.47 \mathrm{E}-03$ & $3.49 \mathrm{E}-04$ & $1.94 \mathrm{E}-04$ \\
\hline${ }^{30} \mathrm{Si}$ & $2.04 \mathrm{E}-03$ & $9.88 \mathrm{E}-04$ & $7.45 \mathrm{E}-03$ & $2.83 \mathrm{E}-03$ & $4.88 \mathrm{E}-04$ & $1.87 \mathrm{E}-04$ \\
\hline${ }^{31} \mathrm{P}$ & $3.86 \mathrm{E}-04$ & $1.91 \mathrm{E}-04$ & $1.36 \mathrm{E}-03$ & $5.91 \mathrm{E}-04$ & $1.34 \mathrm{E}-04$ & $8.34 \mathrm{E}-05$ \\
\hline${ }^{32} \mathrm{~S}$ & $7.74 \mathrm{E}-02$ & $1.87 \mathrm{E}-02$ & $1.07 \mathrm{E}-01$ & $1.21 \mathrm{E}-01$ & $1.27 \mathrm{E}-02$ & $5.76 \mathrm{E}-03$ \\
\hline${ }^{33} \mathrm{~S}$ & $3.62 \mathrm{E}-04$ & $1.01 \mathrm{E}-04$ & $1.04 \mathrm{E}-03$ & $5.18 \mathrm{E}-04$ & $7.71 \mathrm{E}-05$ & $6.36 \mathrm{E}-05$ \\
\hline${ }^{34} \mathrm{~S}$ & $1.96 \mathrm{E}-03$ & $9.80 \mathrm{E}-04$ & $1.11 \mathrm{E}-02$ & $5.65 \mathrm{E}-03$ & $5.50 \mathrm{E}-04$ & $2.23 \mathrm{E}-04$ \\
\hline${ }^{36} \mathrm{~S}$ & $4.10 \mathrm{E}-07$ & $1.49 \mathrm{E}-07$ & $1.30 \mathrm{E}-06$ & $4.78 \mathrm{E}-07$ & $6.19 \mathrm{E}-08$ & $1.97 \mathrm{E}-08$ \\
\hline${ }^{35} \mathrm{Cl}$ & $1.10 \mathrm{E}-04$ & $3.41 \mathrm{E}-05$ & $2.90 \mathrm{E}-04$ & $1.70 \mathrm{E}-04$ & $4.96 \mathrm{E}-05$ & $5.87 \mathrm{E}-05$ \\
\hline${ }^{37} \mathrm{Cl}$ & $2.81 \mathrm{E}-05$ & $7.45 \mathrm{E}-06$ & $7.87 \mathrm{E}-05$ & $5.03 \mathrm{E}-05$ & $8.54 \mathrm{E}-06$ & $1.96 \mathrm{E}-05$ \\
\hline${ }^{36} \mathrm{Ar}$ & $1.21 \mathrm{E}-02$ & $2.54 \mathrm{E}-03$ & $1.21 \mathrm{E}-02$ & $1.93 \mathrm{E}-02$ & $2.19 \mathrm{E}-03$ & $1.49 \mathrm{E}-03$ \\
\hline${ }^{38} \mathrm{Ar}$ & $9.46 \mathrm{E}-04$ & $3.98 \mathrm{E}-04$ & $4.02 \mathrm{E}-03$ & $2.67 \mathrm{E}-03$ & $2.15 \mathrm{E}-04$ & $1.20 \mathrm{E}-04$ \\
\hline${ }^{40} \mathrm{Ar}$ & $6.10 \mathrm{E}-09$ & $1.32 \mathrm{E}-09$ & $1.46 \mathrm{E}-08$ & $5.49 \mathrm{E}-09$ & $7.76 \mathrm{E}-10$ & $8.48 \mathrm{E}-10$ \\
\hline${ }^{39} \mathrm{~K}$ & $6.01 \mathrm{E}-05$ & $1.71 \mathrm{E}-05$ & $1.60 \mathrm{E}-04$ & $1.22 \mathrm{E}-04$ & $3.07 \mathrm{E}-05$ & $6.43 \mathrm{E}-05$ \\
\hline${ }^{40} \mathrm{~K}$ & $4.34 \mathrm{E}-08$ & 7.17E-09 & $7.75 \mathrm{E}-08$ & $3.88 \mathrm{E}-08$ & $1.71 \mathrm{E}-08$ & $1.95 \mathrm{E}-07$ \\
\hline${ }^{41} \mathrm{~K}$ & $4.36 \mathrm{E}-06$ & $1.10 \mathrm{E}-06$ & $1.18 \mathrm{E}-05$ & $8.74 \mathrm{E}-06$ & $1.79 \mathrm{E}-06$ & $1.66 \mathrm{E}-05$ \\
\hline${ }^{40} \mathrm{Ca}$ & $9.73 \mathrm{E}-03$ & $2.04 \mathrm{E}-03$ & $6.82 \mathrm{E}-03$ & $1.69 \mathrm{E}-02$ & $1.71 \mathrm{E}-03$ & $1.52 \mathrm{E}-03$ \\
\hline${ }^{42} \mathrm{Ca}$ & $2.38 \mathrm{E}-05$ & $8.38 \mathrm{E}-06$ & $8.71 \mathrm{E}-05$ & $6.15 \mathrm{E}-05$ & $5.73 \mathrm{E}-06$ & $7.91 \mathrm{E}-06$ \\
\hline${ }^{43} \mathrm{Ca}$ & $7.27 \mathrm{E}-08$ & $1.84 \mathrm{E}-08$ & $1.31 \mathrm{E}-07$ & $1.61 \mathrm{E}-07$ & $5.11 \mathrm{E}-08$ & $3.38 \mathrm{E}-06$ \\
\hline${ }^{44} \mathrm{Ca}$ & $8.40 \mathrm{E}-06$ & $1.94 \mathrm{E}-06$ & $3.31 \mathrm{E}-06$ & $1.59 \mathrm{E}-05$ & $5.66 \mathrm{E}-06$ & $6.22 \mathrm{E}-05$ \\
\hline${ }^{46} \mathrm{Ca}$ & $3.65 \mathrm{E}-11$ & $2.45 \mathrm{E}-12$ & $5.80 \mathrm{E}-11$ & $2.30 \mathrm{E}-11$ & $1.80 \mathrm{E}-12$ & $4.32 \mathrm{E}-10$ \\
\hline${ }^{48} \mathrm{Ca}$ & $2.82 \mathrm{E}-13$ & $1.94 \mathrm{E}-17$ & $4.27 \mathrm{E}-16$ & $1.75 \mathrm{E}-16$ & $2.44 \mathrm{E}-16$ & $3.70 \mathrm{E}-12$ \\
\hline${ }^{45} \mathrm{Sc}$ & $1.33 \mathrm{E}-07$ & $2.55 \mathrm{E}-08$ & $2.38 \mathrm{E}-07$ & $2.29 \mathrm{E}-07$ & $1.59 \mathrm{E}-07$ & $1.59 \mathrm{E}-05$ \\
\hline${ }^{46} \mathrm{Ti}$ & $1.16 \mathrm{E}-05$ & $3.57 \mathrm{E}-06$ & $3.34 \mathrm{E}-05$ & $2.52 \mathrm{E}-05$ & $3.32 \mathrm{E}-06$ & $5.78 \mathrm{E}-05$ \\
\hline${ }^{47} \mathrm{Ti}$ & $5.15 \mathrm{E}-07$ & $1.36 \mathrm{E}-07$ & $7.98 \mathrm{E}-07$ & $1.08 \mathrm{E}-06$ & $8.80 \mathrm{E}-07$ & $8.15 \mathrm{E}-05$ \\
\hline${ }^{48} \mathrm{Ti}$ & $3.65 \mathrm{E}-04$ & $9.23 \mathrm{E}-05$ & $1.65 \mathrm{E}-04$ & $8.16 \mathrm{E}-04$ & $1.38 \mathrm{E}-04$ & $1.34 \mathrm{E}-03$ \\
\hline${ }^{49} \mathrm{Ti}$ & $1.48 \mathrm{E}-05$ & $3.68 \mathrm{E}-06$ & $8.52 \mathrm{E}-06$ & $2.95 \mathrm{E}-05$ & $1.12 \mathrm{E}-05$ & $4.97 \mathrm{E}-04$ \\
\hline${ }^{50} \mathrm{Ti}$ & $1.85 \mathrm{E}-06$ & $9.98 \mathrm{E}-10$ & $3.77 \mathrm{E}-09$ & $1.34 \mathrm{E}-08$ & $2.85 \mathrm{E}-08$ & $7.73 \mathrm{E}-07$ \\
\hline${ }^{50} \mathrm{~V}$ & $3.50 \mathrm{E}-09$ & $3.82 \mathrm{E}-09$ & 7.13E-09 & $4.88 \mathrm{E}-09$ & $6.24 \mathrm{E}-08$ & $4.99 \mathrm{E}-06$ \\
\hline${ }^{51} \mathrm{~V}$ & $4.04 \mathrm{E}-05$ & $2.15 \mathrm{E}-05$ & $2.95 \mathrm{E}-05$ & $6.52 \mathrm{E}-05$ & $1.28 \mathrm{E}-04$ & $3.01 \mathrm{E}-03$ \\
\hline${ }^{50} \mathrm{Cr}$ & 2.61E-04 & $1.56 \mathrm{E}-04$ & $3.15 \mathrm{E}-04$ & $3.84 \mathrm{E}-04$ & $2.03 \mathrm{E}-04$ & $2.42 \mathrm{E}-03$ \\
\hline${ }^{52} \mathrm{Cr}$ & $4.32 \mathrm{E}-03$ & $2.70 \mathrm{E}-03$ & $2.60 \mathrm{E}-03$ & $8.91 \mathrm{E}-03$ & $2.92 \mathrm{E}-03$ & $9.14 \mathrm{E}-03$ \\
\hline${ }^{53} \mathrm{Cr}$ & $6.60 \mathrm{E}-04$ & $7.12 \mathrm{E}-04$ & $6.03 \mathrm{E}-04$ & $1.08 \mathrm{E}-03$ & $1.28 \mathrm{E}-03$ & $9.12 \mathrm{E}-03$ \\
\hline${ }^{54} \mathrm{Cr}$ & $3.48 \mathrm{E}-05$ & $6.85 \mathrm{E}-08$ & $1.82 \mathrm{E}-07$ & $6.68 \mathrm{E}-07$ & $1.17 \mathrm{E}-06$ & $1.07 \mathrm{E}-05$ \\
\hline${ }^{55} \mathrm{Mn}$ & $6.72 \mathrm{E}-03$ & $7.35 \mathrm{E}-03$ & $5.95 \mathrm{E}-03$ & $7.34 \mathrm{E}-03$ & $1.83 \mathrm{E}-02$ & $4.42 \mathrm{E}-02$ \\
\hline${ }^{54} \mathrm{Fe}$ & 7.77E-02 & $8.15 \mathrm{E}-02$ & 7.01E-02 & 7.40E-02 & $5.98 \mathrm{E}-02$ & $6.55 \mathrm{E}-02$ \\
\hline${ }^{56} \mathrm{Fe}$ & $6.57 \mathrm{E}-01$ & $2.95 \mathrm{E}-01$ & $2.78 \mathrm{E}-01$ & $5.59 \mathrm{E}-01$ & $2.57 \mathrm{E}-01$ & $1.11 \mathrm{E}-01$ \\
\hline${ }^{57} \mathrm{Fe}$ & $2.56 \mathrm{E}-02$ & $1.26 \mathrm{E}-02$ & $1.23 \mathrm{E}-02$ & $1.80 \mathrm{E}-02$ & $1.93 \mathrm{E}-02$ & $2.91 \mathrm{E}-02$ \\
\hline${ }^{58} \mathrm{Fe}$ & $2.29 \mathrm{E}-04$ & $1.36 \mathrm{E}-05$ & $1.27 \mathrm{E}-05$ & $1.57 \mathrm{E}-05$ & $6.42 \mathrm{E}-05$ & 3.63E-04 \\
\hline${ }^{59} \mathrm{Co}$ & $8.29 \mathrm{E}-04$ & $8.20 \mathrm{E}-04$ & $6.70 \mathrm{E}-04$ & $6.36 \mathrm{E}-04$ & $1.31 \mathrm{E}-03$ & $3.22 \mathrm{E}-03$ \\
\hline${ }^{58} \mathrm{Ni}$ & $1.06 \mathrm{E}-01$ & $7.24 \mathrm{E}-02$ & $6.63 \mathrm{E}-02$ & 7.03E-02 & $6.19 \mathrm{E}-02$ & $3.90 \mathrm{E}-02$ \\
\hline${ }^{60} \mathrm{Ni}$ & $9.75 \mathrm{E}-03$ & $1.11 \mathrm{E}-02$ & $8.05 \mathrm{E}-03$ & $8.35 \mathrm{E}-03$ & $6.77 \mathrm{E}-03$ & $3.68 \mathrm{E}-03$ \\
\hline${ }^{61} \mathrm{Ni}$ & $2.73 \mathrm{E}-04$ & $7.20 \mathrm{E}-05$ & $6.95 \mathrm{E}-05$ & $2.05 \mathrm{E}-04$ & $4.92 \mathrm{E}-05$ & $2.30 \mathrm{E}-04$ \\
\hline${ }^{62} \mathrm{Ni}$ & $2.52 \mathrm{E}-03$ & $6.35 \mathrm{E}-04$ & $5.85 \mathrm{E}-04$ & $1.61 \mathrm{E}-03$ & $7.41 \mathrm{E}-05$ & $1.07 \mathrm{E}-04$ \\
\hline${ }^{64} \mathrm{Ni}$ & $2.20 \mathrm{E}-07$ & $6.32 \mathrm{E}-11$ & $1.52 \mathrm{E}-10$ & $1.32 \mathrm{E}-09$ & $7.15 \mathrm{E}-09$ & $3.61 \mathrm{E}-07$ \\
\hline${ }^{63} \mathrm{Cu}$ & $1.77 \mathrm{E}-06$ & $6.49 \mathrm{E}-07$ & $5.99 \mathrm{E}-07$ & $1.34 \mathrm{E}-06$ & $1.36 \mathrm{E}-06$ & $1.21 \mathrm{E}-05$ \\
\hline${ }^{65} \mathrm{Cu}$ & $7.78 \mathrm{E}-07$ & $1.96 \mathrm{E}-07$ & $1.82 \mathrm{E}-07$ & $7.85 \mathrm{E}-07$ & $1.94 \mathrm{E}-08$ & $5.56 \mathrm{E}-07$ \\
\hline${ }^{64} \mathrm{Zn}$ & $1.43 \mathrm{E}-05$ & $3.74 \mathrm{E}-06$ & $3.23 \mathrm{E}-06$ & $1.38 \mathrm{E}-05$ & $9.80 \mathrm{E}-07$ & $5.11 \mathrm{E}-06$ \\
\hline${ }^{66} \mathrm{Zn}$ & $2.37 \mathrm{E}-05$ & $6.39 \mathrm{E}-06$ & $5.58 \mathrm{E}-06$ & $2.13 \mathrm{E}-05$ & $1.02 \mathrm{E}-07$ & $3.36 \mathrm{E}-07$ \\
\hline${ }^{67} \mathrm{Zn}$ & $4.94 \mathrm{E}-11$ & $2.29 \mathrm{E}-11$ & $2.28 \mathrm{E}-11$ & $8.14 \mathrm{E}-11$ & $2.23 \mathrm{E}-10$ & $2.32 \mathrm{E}-08$ \\
\hline${ }^{68} \mathrm{Zn}$ & $9.59 \mathrm{E}-09$ & $2.71 \mathrm{E}-09$ & $2.44 \mathrm{E}-09$ & $9.55 \mathrm{E}-09$ & $2.36 \mathrm{E}-10$ & $6.69 \mathrm{E}-09$ \\
\hline${ }^{70} \mathrm{Zn}$ & $2.36 \mathrm{E}-14$ & $2.60 \mathrm{E}-19$ & $2.89 \mathrm{E}-18$ & $5.53 \mathrm{E}-17$ & $5.78 \mathrm{E}-15$ & $2.93 \mathrm{E}-11$ \\
\hline
\end{tabular}


TABLE 4

Masses of Radioactive SPECIES $\left(\mathrm{M}_{\odot}\right)$ Before RADIOACTIVE DECAYS.

\begin{tabular}{ccccc}
\hline \hline Isotopes & W7 & C-DEF & C-DDT & O-DDT \\
\hline${ }^{22} \mathrm{Na}$ & $2.01 \mathrm{E}-08$ & $1.01 \mathrm{E}-07$ & $1.46 \mathrm{E}-07$ & $5.40 \mathrm{E}-08$ \\
${ }^{26} \mathrm{Al}$ & $5.18 \mathrm{E}-07$ & $1.69 \mathrm{E}-06$ & $2.47 \mathrm{E}-06$ & $8.77 \mathrm{E}-07$ \\
${ }^{36} \mathrm{Cl}$ & $2.08 \mathrm{E}-06$ & $4.74 \mathrm{E}-07$ & $5.22 \mathrm{E}-06$ & $2.06 \mathrm{E}-06$ \\
${ }^{39} \mathrm{Ar}$ & $6.79 \mathrm{E}-09$ & $1.53 \mathrm{E}-09$ & $1.69 \mathrm{E}-08$ & $7.75 \mathrm{E}-09$ \\
${ }^{40} \mathrm{~K}$ & $4.34 \mathrm{E}-08$ & $7.17 \mathrm{E}-09$ & $7.75 \mathrm{E}-08$ & $3.90 \mathrm{E}-08$ \\
${ }^{41} \mathrm{Ca}$ & $4.35 \mathrm{E}-06$ & $1.10 \mathrm{E}-06$ & $1.18 \mathrm{E}-05$ & $8.85 \mathrm{E}-06$ \\
${ }^{44} \mathrm{Ti}$ & $8.37 \mathrm{E}-06$ & $1.93 \mathrm{E}-06$ & $3.21 \mathrm{E}-06$ & $1.59 \mathrm{E}-05$ \\
${ }^{48} \mathrm{~V}$ & $4.32 \mathrm{E}-08$ & $1.68 \mathrm{E}-08$ & $9.76 \mathrm{E}-08$ & $1.09 \mathrm{E}-07$ \\
${ }^{49} \mathrm{~V}$ & $1.05 \mathrm{E}-07$ & $1.00 \mathrm{E}-07$ & $3.07 \mathrm{E}-07$ & $2.69 \mathrm{E}-07$ \\
${ }^{53} \mathrm{Mn}$ & $1.64 \mathrm{E}-04$ & $4.93 \mathrm{E}-04$ & $3.38 \mathrm{E}-04$ & $2.25 \mathrm{E}-04$ \\
${ }^{55} \mathrm{Fe}$ & $1.79 \mathrm{E}-03$ & $4.17 \mathrm{E}-03$ & $2.89 \mathrm{E}-03$ & $1.93 \mathrm{E}-03$ \\
${ }^{60} \mathrm{Fe}$ & $3.33 \mathrm{E}-09$ & $9.86 \mathrm{E}-15$ & $2.29 \mathrm{E}-13$ & $6.93 \mathrm{E}-12$ \\
${ }^{55} \mathrm{Co}$ & $4.89 \mathrm{E}-03$ & $3.18 \mathrm{E}-03$ & $3.07 \mathrm{E}-03$ & $5.40 \mathrm{E}-03$ \\
${ }^{56} \mathrm{Co}$ & $1.21 \mathrm{E}-04$ & $1.18 \mathrm{E}-04$ & $1.06 \mathrm{E}-04$ & $1.04 \mathrm{E}-04$ \\
${ }^{57} \mathrm{Co}$ & $9.52 \mathrm{E}-04$ & $1.94 \mathrm{E}-03$ & $1.40 \mathrm{E}-03$ & $9.37 \mathrm{E}-04$ \\
${ }^{60} \mathrm{Co}$ & $4.32 \mathrm{E}-08$ & $5.30 \mathrm{E}-10$ & $1.19 \mathrm{E}-09$ & $3.30 \mathrm{E}-09$ \\
${ }^{56} \mathrm{Ni}$ & $6.40 \mathrm{E}-01$ & $2.45 \mathrm{E}-01$ & $2.46 \mathrm{E}-01$ & $5.40 \mathrm{E}-01$ \\
${ }^{57} \mathrm{Ni}$ & $2.46 \mathrm{E}-02$ & $1.06 \mathrm{E}-02$ & $1.09 \mathrm{E}-02$ & $1.71 \mathrm{E}-02$ \\
${ }^{59} \mathrm{Ni}$ & $4.66 \mathrm{E}-04$ & $7.24 \mathrm{E}-04$ & $5.78 \mathrm{E}-04$ & $4.22 \mathrm{E}-04$ \\
${ }^{63} \mathrm{Ni}$ & $4.82 \mathrm{E}-08$ & $4.28 \mathrm{E}-11$ & $1.85 \mathrm{E}-10$ & $1.22 \mathrm{E}-09$ \\
& & & &
\end{tabular}

$Y_{\mathrm{e}}=0.5$, which should introduce some errors when the electron capture reactions are active. This could to some extent affect the nucleosynthesis in the initial deflagration phase $(\S 3.4)$, and therefore the ratio ${ }^{58} \mathrm{Ni} /{ }^{56} \mathrm{Fe}$.

The intermediate mass elements can also be used to constrain SN Ia models. Assuming a typical fraction of $\sim 20 \%$ for the frequency of SNe Ia as compared to core-collapse $\mathrm{SNe}$, then $(\mathrm{Si} / \mathrm{Fe}) /(\mathrm{Si} / \mathrm{Fe}) \odot \lesssim 0.5$ is required to compensate the over-solar production in core-collapse SNe. The O-DDT model has the ratio $(\mathrm{Si} / \mathrm{Fe}) /(\mathrm{Si} / \mathrm{Fe})_{\odot} \sim 1$; thus, the model can explain at most a half of SNe Ia. The C-DDT is not at all favored, since $(\mathrm{Si} / \mathrm{Fe}) /(\mathrm{Si} / \mathrm{Fe}) \odot \sim 2$, i.e., it produces too much IMEs relative to Fe. The deflagration models (W7 and $\mathrm{C}-\mathrm{DEF}$ ) do not have the problem in the $\mathrm{Si} / \mathrm{Fe}$ ratio $\left[(\mathrm{Si} / \mathrm{Fe}) /(\mathrm{Si} / \mathrm{Fe})_{]}<0.5\right]$. In the O-DDT model, all the elements heavier than $\mathrm{N}$ are consistent with $\mathrm{W} 7$ within a factor of two.

In reality, the solar abundances are the superposition of contributions from $\mathrm{SNe}$ at various metallicities, and we expect that the "average" SNe Ia to occur at a metallicity less than solar. We indeed expect some improvement if we consider a lower metallicity (see e.g., Travaglio et al. 2005): a decreased metallicity should result in a smaller ${ }^{58} \mathrm{Ni} /{ }^{56} \mathrm{Fe}$ ratio in the delayed detonation models. Also, the smaller metallicity is expected to lead to the smaller amount of ${ }^{54} \mathrm{Fe} . \mathrm{Mg}$ and $\mathrm{Al}$ are also affected: If the metallicity is $10 \%$ of the solar value, then the amount of ${ }^{24} \mathrm{Mg}$ could increase by $\sim 50 \%$, and that of ${ }^{27} \mathrm{Al}$ could decrease by a factor of a few. These changes do not conflict with the Galactic chemical evolution, since the ratios $(\mathrm{Mg}, \mathrm{Al}) / \mathrm{Fe}$ are much smaller than the solar values in the O-DDT models.

Summarizing, the hypothesis that about half of $\mathrm{SNe}$ Ia are represented by the extreme O-DDT model, is not rejected by the chemical evolution arguments. Note that this should also apply to globally symmetric, but offcenter, delayed detonation models to some extent, as these models should share the basic feature that the cen- tral region is burned to Fe-peak and ${ }^{56} \mathrm{Ni}$ by the detonation $(\S 4)$. Therefore, multi-D delayed detonation models can potentially account for a majority of SNe Ia.

\section{CONCLUDING REMARKS}

We have presented results of the detailed nucleosynthesis calculations for 2D delayed detonation models, focusing on an extremely off-center model (O-DDT model). Features are different from classical 1D models. Unlike a globally symmetric delayed detonation model following the central ignition of the deflagration flame (C-DDT model), the detonation wave proceeds both in the high density region near the center of a white dwarf and in the low density region near the surface. Thus, the resulting yield is a mixture of different explosive burning products, from carbon-burning at low densities to complete silicon-burning at the highest densities, as well as the electron-capture products from the deflagration stage.

The evolution of the deflagration flame can be different in 2D and in 3D simulations (e.g., Röpke et al. 2007b). We believe that the global feature found in this study, i.e., the detonation wave propagating into the innermost region, would not be changed substantially in 3D simulations. However, some details would be affected: For example, 3D simulations tend to result in stronger deflagration than in 2D simulations for similar initial conditions (e.g., Travaglio et al. 2004a). As the stronger deflagration is followed by the weaker detonation in our DDT models, this would result in a shift of the overall energetic and the nucleosynthesis production as compared to the 2D models. On the other hand, although in our 2D simulations the detonation wave has to burn around the deflagration ash blob before reaching to the center, the deflagration ash blob may have holes, depending on the ignition geometry through which the detonation wave can directly penetrate into the center in 3D simulations. Thus, future 3D simulations are important to obtain the robust model predictions as a function of the model input (e.g., distribution of the deflagration ignition sparks).

The yield of the O-DDT model largely satisfies constraints from the Galactic chemical evolution despite the low DDT density as compared to 1D delayed detonation models. This is a result of qualitatively different behavior of the detonation propagation in $1 \mathrm{D}$ and multi-D models; the outward propagation in former (which is also the case in the C-DDT model), while the inward propagation in the latter.

The O-DDT model could thus account for a fraction of SNe Ia (especially bright ones). As less-extreme (more spherical) models are expected to share the common properties in the integrated yield, the multi-D delayed detonation model could potentially account for a main population of SNe Ia.

The delayed detonation models also provide a characteristic layered structure, unlike multi-dimensional, especially $2 \mathrm{D}$, deflagration models ${ }^{7}$. In the O-DDT model, the region filled by electron capture species (e.g., ${ }^{58} \mathrm{Ni}$, ${ }^{54} \mathrm{Fe}$ ) shows a large off-set, and the region is within a shell above the bulk of the ${ }^{56} \mathrm{Ni}$ distribution near the center. These can be directly tested by observations.

We note that the distribution of the nucleosynthsis

7 Note that the 3D deflagration models can also potentially produce the layered structure (Röpke et al. 2007b). 
products in our 2D DDT models is somewhat different from the result of Bravo \& García-Senz (2008). Their 3D DDT models lack a clear abundance stratification, and they are characterized by large mass fractions of Fepeak elements near the surface regions. In contrast, the 3D DDT models of Röpke \& Niemeyer (2007c), do produce a layered structure with the surface dominated by IMEs - similar to our present 2D models (§3.5). It seems that (1) the deflagration is stronger in Bravo \& GarcíaSent (2008) than in our 2D models and in 3D models of Röpke \& Niemeyer (2007c), and (2) the detonation wave is mainly propagating inward in Bravo \& García-Senz (2008) although it is propagating both inward (producing Fe-peak elements) and outward (producing IME) in our simulations. As a result, the amount of unburned material is smaller in our models. The cause of the different flame propagation is not clear, but likely due to different treatment of thermonuclear flames. The overall abundance distribution of the 3D DDT models of Gamezo et al. (2005), on the other hand, is similar to our 2D models, producing the stratified configuration. In their simulations, they initiated the detonation from the center at relatively high DDT density, and the detonation wave propagates outward, producing the layered composition structure as the temperature drops following the detonation propagation. This is, indeed, quite different from our models, in which the detonation is initiated at relatively low DDT density but propagates inward to the high density central region.

The observational consequences from similar models have been discussed by Kasen et al. (2009) for the early photospheric phase (see also Hillebrandt et al. 2007; Sim et al. 2007). Here, we summarize some additional, expected observational characteristics, especially in the late-time nebular phase (taken after a few hundred days). We emphasize that the late-time spectroscopy is currently the most effective way to hunt for the signature of the DDT model in the innermost region. See also Maeda et al. (2010) who discussed the following points in details.

- Abundance Stratification and carbon near the surface: Overall, the stratified composition structure obtained in our 2D models is consistent with the result of the "abundance tomography" (Stehle et al. 2005; Mazzali et al. 2008). Spectrum synthesis models as compared to the observed early-phase photospheric spectra show that the mass fraction of carbon at $\sim 10,000-14,000$ $\mathrm{km} \mathrm{s}^{-1}$ should be smaller than 0.01 (e.g., Branch et al. 2003; Thomas et al. 2007; Tanaka et al. 2008). This constraint is satisfied by our DDT models at $10,000 \mathrm{~km} \mathrm{~s}^{-1}$, although it is marginal at 14,000 $\mathrm{km} \mathrm{s}^{-1}$. The latter could, however, be improved by changing the DDT criterion such that the transition on average proceeds at higher densities (e.g., Iwamoto et al. 1999). Another interesting observational target is the inhomogeneous distribution of the unburned $\mathrm{C}+\mathrm{O}$ pockets near the surface both in C-DDT and O-DDT models (§3.5), although the total amount of such unburned material is small in the DDT models. Detectable carbon absorption lines may appear only when the observed line-ofsight intersects a large number of such unburned pockets, which may explain the low frequency of $\mathrm{SNe}$ Ia showing the $\mathrm{C}$ absorption lines detected (e.g., Tanaka et al. 2006).

- [O I] $\lambda \lambda 6 \mathbf{6 3 0 0 , 6 3 6 3 :}$ The main problem in the pure deflagration model is existence of unburned carbon and oxygen mixed down to the central region. This should produce a strong [O I] $\lambda \lambda 6300$, 6363 doublet in late-time spectra, although no such signature has been detected in the observations (Kozma et al. 2005). This tension is alleviated in 3D deflagration models, with the mass fraction of unburned elements going down to $10 \%$ (Röpke et al. $2007 \mathrm{~b}$ ) or even to smaller values (e.g., Travaglio et al. 2004a). The DDT model does not have this problem, as unburned material near the center is burned in the subsequent detonation. A detailed study of late-time nebular spectra should provide us with information on this issue.

- Line shifts: Profiles of nebular emission lines can be used to effectively trace the distribution of the burning products, as is proven to be efficient for core-collapse SNe (Maeda et al. 2002; Mazzali et al. 2005; Maeda et al. 2008; Modjaz et al. 2008; Taubenberger et al. 2009). The O-DDT model predicts that (1) the distribution of stable Fe-peak elements is off-set following the asymmetric deflagration flame propagation, while the detonation products (e.g., a large fraction of ${ }^{56} \mathrm{Ni}$ ) are distributed more or less in a spherical manner (Figs. 5 and 12). Recently, Maeda et al. (2010) found that the expected variation of the line wavelength is seen in nebular spectra of SNe Ia, indicating that the above configuration can be relatively common in SNe Ia.

- Line profiles: Detailed profiles of the nebular emission lines can be used to infer the distribution of emitting ions. It has been suggested to use NearInfrared (NIR) spectroscopy to hunt for the geometry, as NIR [Fe II] lines are not severely blended (e.g., Höflich et al. 2004; Motohara et al. 2006). This can also be done using several IR lines like [Co III $] 11.88 \mu \mathrm{m}$ (Gerardy et al. 2007). The best data to date have been obtained for SN 2003hv, showing a hole in the distribution of ${ }^{56} \mathrm{Ni}^{8}$. Because the mixing is introduced by the deflagration, such a hole is difficult to understand in the present models (and most of SN Ia explosion models; but see Meakin et al. 2009). This issue remains unresolved, and need further study preferentially in 3D simulation.

We would like to thank Ken'ichi Nomoto for kindly providing the thermal history of the W7 model. This research has been supported by World Premier International Research Center Initiative (WPI Initiative), MEXT, Japan. The work of K.M. is also supported through the Grant-in-Aid for Young Scientists

\footnotetext{
8 Note, however, that the central wavelengths of the emission lines are shifted with respective to the explosion center, and this "line shift" can be well explained by the off-set DDT scenario (Maeda et al. 2010).
} 
(20840007) of Japanese Society for Promotion of Science (JSPS). The work of F.K.R. is supported through the Emmy Noether Program of the German Research Foundation (DFG; RO 3676/1-1) and by the Cluster of Excellence "Origin and Structure of the Universe" (EXC 153).
The work by F.-K.T. is supported by the Swiss National Science Foundation(SNF) and the Alexander von Humholdt Foundation. The calculations have been performed on IBM Power5 system at Rechenzentrum Garching (RZG) of the Max-Planck Society.

\section{REFERENCES}

Arnett, W.D. 1969, Ap\&SS, 5, 180

Arnett, W.D. 1996, Supernovae and Nucleosynthesis (Princeton University Press)

Brachwitz, F., Dean, D.J., Hix, W.R., Iwamoto, K., Langanke, K., Martinez-Pinedo, G., Nomoto, K., Strayer, M.R., Thielemann, F.-K., \& Umeda, H. 2000, ApJ, 536, 934

Branch, D., Doggett, J.B., Nomoto, K., \& Thielemann, F.-K. 1985, ApJ, 294, 619

Branch, D. 1998, ARA\&A, 36, 17

Branch, D., et al. 2003, AJ, 126, 1489

Bravo, E., \& García-Senz, D. 2006, ApJ, 642, L157

Bravo, E., \& García-Senz, D. 2008, A\&A, 478, 843

Fink, M., Hillebrandt, W., \& Röpke, F. 2007, A\&A, 476, 1133

Fuller, G.M.,Fowler, W.A., \& Newman, M. 1982, ApJS, 48, 279

Fuller, G.M.,Fowler, W.A., \& Newman, M. 1985, ApJ, 293, 1

Gamezo, V.N., Khokhlov, A.M., Oran, E.S., Chtchelkanova, A.Y., \& Rosenberg, R.O. 2003, Science, 299, 77

Gamezo, V.N., Khokhlov, A.M., Oran, E.S. 2005, ApJ, 623, 337

Gerardy, C.L., et al. 2007, ApJ, 661, 995

Golombek, I., \& Niemeyer, J.C. 2005, A\&A 438, 611

Goswami, A., \& Prantzos, N. 2000, A\&A, 359, 191

Hillebrandt, W., Sim, S. A., \& Röpke, F. 2007, A\&A, 465, 17

Höflich, P., \& Khokhlov, A. 1996, ApJ, 457, 500

Höflich, P., et al. 2004, ApJ, 617, 1258

Iben, I. Jr., \& Tutukov, A.V. 1984, ApJS, 54, 335

Iwamoto, K., Brachwitz, F., Nomoto, K., Kishimoto, N., Umeda H., Hix, W.R., \& Thielemann, F.-K. 1999, ApJS, 125, 439

Jordan, G.C., et al. 2008, ApJ, 681, 1448

Kasen, D., Röpke, F.K., \& Woosley, S.E. 2009, Nature, 460, 869

Khokhlov, A., 1991, A\&A, 245, 114

Kozma, C., Fransson, C., Hillebrandt, W., Travaglio, C., Sollerman, J., Reinecke, M., Röpke, F.K., \& Spyromilio, J. 2005, A\&A, 437, 983

Kuhlen, M., Woosley, S.E., \& Glatzmaier, G.A. 2006, ApJ, 640, 407

Langanke, K., \& Martinez-Pinedo, G. 2000, Nucl. Phys. A, 673 481

Maeda, K., Nakamura, T., Nomoto, K., Mazzali, P.A., Patat, F., \& Hachisu, I. 2002, ApJ, 565, 405

Maeda, K., \& Nomoto, K. 2003, ApJ, 598, 1163

Maeda, K., et al. 2008, Science, 319, 1220

Maeda, K., Taubenberger, S., Sollerman, J., Mazzali, P.A., Leloudas, G., Nomoto, K., \& Motohara, K. 2010, ApJ, 708, 1703 Maier, A. \& Niemeyer, J.C. 2006, A\&A, 451, 207

Martinez-Pinedo, G., Langanke, K., \& Dean, D.J., 2000, ApJS, 126,493

Mazzali, P.A., et al. 2005, Science, 308, 1284

Mazzali, P.A., Röpke, F.K., Benetti, S., \& Hillebrandt, W. 2007 Science, 315,825

Mazzali, P.A., Sauer, D.N., Pastorello, A., Benetti, S., Hillebrandt, W. 2008, MNRAS, 386, 1897

Meakin, C.A., Seitenzahl, I., Townsley, D., Jordan, G.C., Truran, J., Lamb, D. 2009, ApJ, 693, 1188

Modjaz, M., Kirshner, R.P., Blondin, S., Challis, P., \& Matheson, T. 2008, ApJ, 687, L9

Motohara, K., et al. 2006, ApJ, 652, L101

Nagataki, S., Hashimoto, M., Sato K., \& Yamada, S. 1997, ApJ, 486, 1026

Niemeyer, J.C., Hillebrandt, W., \& Woosley, S.E. 1996, ApJ, 471, 903

Nomoto, K. 1982, ApJ, 253, 798

Nomoto, K., Thielemann, F.-K., \& Yokoi, K. 1984, ApJ, 286, 644
Nomoto, K., Iwamoto, K., \& Kishimoto, N. 1997, Science, 276, 1378

Nugent, P., Baron, E., Branch, D., Fisher, A., \& Hauschildt, P.H. 1997, ApJ, 485, 812

Plewa, T. 2007, ApJ, 657, 942

Reinecke, M., Hillebrandt, W., \& Nimeyer, J.C. 1999a, A\&A, 347, 739

Reinecke, M., Hillebrandt, W., Niemeyer, J. C., Klein, R., Gröbl, A. 1999b, A\&A, 347, 724

Reinecke, M., Hillebrandt, W., \& Nimeyer, J.C. 2002, A\&A, 391, 1167

Röpke, F.K., \& Hillebrandt, W. 2005, A\&A, 431, 635

Röpke, F.K., Gieseler, M., Reinecke, M., Travaglio, C., \& Hillebrandt, W 2006a, A\&A 453, 203

Röpke, F.K., Hillebrandt, W., Niemeyer, J.C., \& Woosley, S.E. 2006b, A\&A, 448, 1

Röpke, F.K. 2007a, ApJ, 668, 1103

Röpke, F.K., Hillebrandt, W., Schmidt, W., Niemeyer, J.C., Blinnikov, S.I., \& Mazzali, P.A. 2007b, ApJ, 668, 1132

Röpke, F.K., \& Niemeyer, J.C. 2007c, A\&A, 464, 683

Röpke, F.K., Woosley, S.E., \& Hillebrandt, W. 2007d, ApJ, 660, 1344

Schmidt, W., \& Nimeyer, J.C. 2006, A\&A, 446, 627

Sim, S. A., Sauer, D. N., Röpke, F. K., \& Hillebrandt, W. 2007, MNRAS, 378, 2

Stehle, M., Mazzali, P.A., Benetti, S., Hillebrandt, W. 2005, MNRAS, 360, 1231

Tanaka, M., Mazzali, P.A., Maeda, K., \& Nomoto, K. 2006, ApJ, 645,470

Tanaka, M., et al. 2008, ApJ, 677, 448

Taubenberger, S., et al. 2009, MNRAS, 397, 677

Thielemann, F.-K., Nomoto, K., \& Yokoi, K 1986, A\&A, 158, 17

Thielemann, F.-K., Nomoto, K., \& Hashimoto, M. 1996, ApJ, 460, 408

Thielemann, F.-K., Rauscher, T., Freiburghaus, C., Nomoto, K., Hashimoto, M., Pfeiffer, B., \& Kratz, K.-L. 1998, Nuclear and Particle Astrophysics (Cambridge University Press; Cambridge) (eds. J. G. Hirsch and D. Page), 27

Thielemann, F.-K., Brachwitz, F., H"oflich, P., Martinez-Pinedo, G., \& Nomoto, K. 2004, New Astronomy Reviews, 48, 605

Thomas, R.C., et al. 2007, ApJ, 654, L53

Travaglio, C., Hillebrandt, W., Reinecke, M., \& Thielemann, F.-K. 2004a, A\&A, 425, 1029

Travaglio, C., Kifonidis, K., \& Müller, E. 2004b, New Astron. Rev., 48,25

Travaglio, C., Hillebrandt, W., \& Reinecke, M. 2005, A\&A, 443, 1007

Webbink, R.F. 1984, ApJ, 277, 355

Wheeler, J.C., Harkness, R.P., Khokhlov, A.M., \& Höflich, P. 1995, Phys. Rep., 53, 221

Whelan, J., \& Iben, I., Jr. 1973, ApJ, 186, 1007

Woosley, S.E., \& Weaver, T.A. 1986, ARA\&A, 24, 205

Woosley, S.E., \& Weaver, T.A. 1994, ApJ, 423, 371

Woosley, S.E., Wunsch, S., \& Kuhlen, M. 2004, ApJ, 607, 921

Woosley, S.E. 2007, ApJ, 668, 1109

Woosley, S.E., Kerstein, A.R., Sankaran, V., \& Röpke, F.K. 2009, ApJ, 704, 255

Yamaoka, H., Shigeyama, T., Nomoto, K., \& Thielemann, F.-K. 1992, ApJ, 393, L55 